\title{
A SINGLE SERVER QUEUE UNDER RANDOM VACATION POLICY
}

\author{
Priyanka Kalita* and Gautam Choudhury
}

\begin{abstract}
This paper deals with an $M / G / 1$ queueing system with random vacation policy, in which the server takes the maximum number of random vacations till it finds minimum one message (customer) waiting in a queue at a vacation completion epoch. If no arrival occurs after completing maximum number of random vacations, the server stays dormant in the system and waits for the upcoming arrival. Here, we obtain steady state queue size distribution at an idle period completion epoch and service completion epoch. We also obtain the steady state system size probabilities and system state probabilities. Some significant measures such as a mean number of customers served during the busy period, Laplace-Stieltjes transform of unfinished work and its corresponding mean value and second moment have been obtained for the system. A cost optimal policy have been developed in terms of the average cost function to determine a locally optimal random vacation policy at a lower cost. Finally, we present various numerical results for the above system performance measures.
\end{abstract}

Mathematics Subject Classification. 60K25, 90B22, 60K20.

Received September 26, 2017. Accepted September 6, 2019.

\section{INTRODUCTION}

Queueing models with server vacations have been effectively studied by many researchers in the last few decades and successfully applied in the various problems such as computer communication networks, modern telecommunication systems, production, manufacturing systems, etc. An excellent survey on the vacation queueing models have been documented in Doshi [5,6], Takagi [26], Tian and Zhang [28] and others. There are two basic vacation queueing models namely, multiple vacation queueing model and single vacation queueing model. In multiple vacation queueing models, the server keeps on taking consecutive vacations until it finds minimum one customer waiting in a queue at a vacation completion epoch; However, in single vacation queueing models, the server takes exactly one vacation between two consecutive busy periods. These two types of vacation models were first introduced by Levy and Yechiali [20].

In this paper, we consider an $M / G / 1$ queueing system with random vacation policy (RVP). When the server finishes the service to all customers and the system becomes empty, the server leaves for a vacation. The server is allowed to take a maximum number, denoted by $Y$, successive vacations if the system remains empty after the end of a vacation. After completing $Y$ successive vacations if the system is still empty, the server becomes dormant in the system and waits for the upcoming arrival. The maximum number of $Y$ vacations taken by the server is a discrete random variable (r.v) with probability mass function (PMF) $P\{Y=j\}=y_{j} ; j=1,2, \ldots$.

Keywords. $M / G / 1$ queue, Random vacation policy, embedded Markov chain, Markov regenerative process, cost optimal policy.

Mathematical Sciences Division, Institute of Advanced Study in Science and Technology, 781035 Guwahati, Assam, India.

*Corresponding author: priya.24723@gmail.com 
There have been several models evolved in recent years for vacation queueing systems with a dormant period. The notion of a dormant period was first introduced by Heyman [8], where he assumed that the dormant period is a period in which the server is waiting for a number of customers. Later, Choudhury [2] studied the dormant period, which arises between vacation period and busy period. Moreover, Ke et al. [11] investigated such a vacation queueing system with dormant period and its application to production and inventory systems.

The optimal control policy has received a great interest in recent years in the vacation queueing systems. The first study of vacation queueing systems with control policy was done by Kella [14]. The variations and expansions of these vacation queueing systems with control policy can be referred to Lee and Srinivasan [16], Lee et al. [17,18], Tadj and Choudhury [24] and others. Further, Ke [10] analyzed the operating characteristic of an $M^{X} / G / 1$ system with multiple variant vacation policy and balking. In the same paper, it is presumed that the server takes at most $Y$ number of vacations repeatedly until it finds atleast one customer at a vacation completion epoch and obtained the steady state system size probabilities and steady state queue size distribution at departure point using the well known supplementary variable technique. Recently, Yang et al. [30] discussed an optimal control policy of an unreliable retrial $M^{X} / G / 1$ system with general repeated attempts and $J$ optional multiple vacations. In this queueing system, when the unreliable server becomes empty, it leaves for the first essential vacation, after which it may either remains idle within the system or take one of $J$ optional vacations. It is also presumed that when the server is busy, it is subject to random breakdowns and repair. They derived the probability generating function (PGF) of the system size distribution at a random epoch, some system performance measures as well as two reliability indices.

Many researchers have generalized multiple and single vacation queueing model due to its numerous applications in production management, computer communication networks, telecommunication systems, etc. Takagi [26] and Leung [19] considered such a model in which they introduced the notion of RVP (a generalization of multiple and single vacation), where the server takes at most $Y$ numbers of random vacations repeatedly until it finds minimum one customer at a vacation completion epoch. If no arrival occurs by the end of the vacation the server becomes idle. A wide class of vacation policies for governing the vacation mechanism has been discussed in the survey of Ke et al. [12]. Moreover, Ke et al. [11] have made an extensive investigation on RVP queueing systems. However, a further generalization of such a model is also possible by considering $Y$ as a r.v instead of a fixed number (i.e., the maximum number of vacations). Yang et al. [31] discussed a discrete-time $G e o / G e o / 1$ queueing system with multiple working vacations (a generalization of multiple vacations). In the same paper, it is presumed that when the system becomes empty, the server enters into a working vacation i.e., during a working vacation the arriving customers are served by a lower service rate. They also investigated customers equilibrium strategies for joining or balking. Later, Jeyakumar and Senthilnathan [9] also studied such type of multiple working vacations but with an unreliable $M^{X} / G / 1$ queueing system. They obtained the PGF the system size distribution at a random epoch as well as other completion epoch. More recently, Ke et al. [13] discussed an $M / M / C$ balking retrial queueing system with both single and multiple vacation policy. In the same paper, some useful formula and various system performance measures are also obtained.

In the literature, the steady state performance measures are studied for various exhaustive service queueing systems with a random vacation policy which focuses on the maximum number of random vacations $Y$, which is a fixed number. However, only a very few works available in the literature concerned with the maximum number of vacations $Y$ is a r.v. So far, to best of our knowledge, Tian [27], Zhang and Tian [32] have investigated such type of model. Although some aspects of random vacation policy (where the maximum number of vacations $Y$ is a r.v) have been studied by these authors but still some questions needed to be addressed. The questions are

- What is the real life application of this type model?

- What is the PGF of queue size distribution at idle period completion epoch?

- What is the steady state equation for queue size distribution at service completion epoch?

- What is the steady state system size probabilities for different state along with closed form expression for global kernel matrix $K(t)$ and local kernel matrix $L(t)$ ? 
- What is the distribution of a number of customers served during the busy period?

- What is the unfinished work and cost optimal policy for this model?

This type of model has a potential application in the IEEE 802.16e system, which supports the mobility of a mobile station (MS) by involving Sleep/Awake period (mode) operational sequence to save the battery power of MS. Here, sleep period is modeled as random vacation period (contains maximum $Y$ number of random vacations), warm-up period is modeled as dormant period and awake period is modeled as busy period. The detailed justification of the proposed model with application is illustrated in Section 2. This motivates us to study such a model in the form of random vacation policy in this paper. Hence, in this paper, we proposed to investigate such a type of $M / G / 1$ queueing system with random vacation policy.

The rest of the paper is arranged as follows: The real world justification of the queueing model under consideration is presented in Section 2. In Section 3, the mathematical model is described. In Section 4, the queue size distribution at idle period completion epoch, at service completion epoch and steady state system size probabilities using embedded Markov chain (EMC) and Markov regenerative process (MRGP) are derived. Some other essential system performance measures of this model are obtained in Section 5. An important particular case of this model is demonstrated in Section 6. Section 7 display the cost optimal policy of our proposed model along with average cost function. Finally, in Section 8, some important numerical results are presented.

\section{REAL WORLD JUSTIFICATION OF OUR PROPOSED MODEL}

In an IEEE802.16e system, power handling is a significant component of mobility support of a mobile station (MS) by involving Sleep/Awake period (mode) operational sequence, see Seo et al. [23]. Sleep period is one of the major important feature for battery power saving in MS to extend their functional life. Sleep period is a state in which MS is unavailable for a period of time, which is observed from serving Base station (BS). Before starting the sleep period, an MS sends a signal to a serving BS and waits for BS's approval. After getting the positive approval (i.e., there is no data to be sent to MS), it begins a sleep period. The sleep period consist of many sleep intervals. The sleep intervals are denoted by $S l$ which is considered to be a generic r.v. After the end of every sleep interval, it wakes up and inspect whether there are any frames or messages (data) for it or not. This task is carried out in the ON interval and is denoted by $T_{o p}$. Here, we are assuming $T_{o p}$ to be a constant. Any arrival during an ON interval can only be notified in the following ON interval. Here, the initial sleep interval start with an ON interval. If no arrival occurs at the end of a sleep interval, it will continue for an upper bound on a number of sleep intervals. On the other hand, if any data arrived to MS during the ON interval (i.e., after the sleep interval), it starts awake period, otherwise it goes for the last ON interval. The last ON interval is included in the warm-up period and is denoted by $T_{w 1}$ (in practice, $T_{o p}=T_{w 1}$ ) (see $[1,23]$ ). Warm-up period is a period where the MS waits for the first arrival after completing a maximum number of sleep.

Figures 1 and 2 show the period (mode) of an $M / G / 1$ system with random vacation policy to the feasible operational mode of an MS during two different regeneration cycles namely, type 1 cycle and type 2 cycle respectively. The regeneration cycle is defined as the elapsed time from the epoch in which a random vacation period is enabled, until the epoch where the next random vacation period is about to begin. Type 1 cycle represent that if any customer arrive during the random vacation period (i.e., sleep period), the server starts service immediately at that random vacation completion epoch, which is shown in Figure 1. On the other hand, type 2 cycle represent that if no arrival occurs during a random vacation period, the server stays dormant (warm-up) in the system and if any arrival occurs during this dormant period, it immediately starts service at that dormant period completion epoch, which is shown in Figure 2.

Seo et al. [23] suggested an $M / G / 1$ model with multiple vacations to evaluate the dropping probability, average waiting time and average delay of the packets in a BS's buffer. Park and Hwang [21] also proposed this type of model, but for finite capacity to analyse the sleep-period operation of a standard IEEE 802.16e system. 


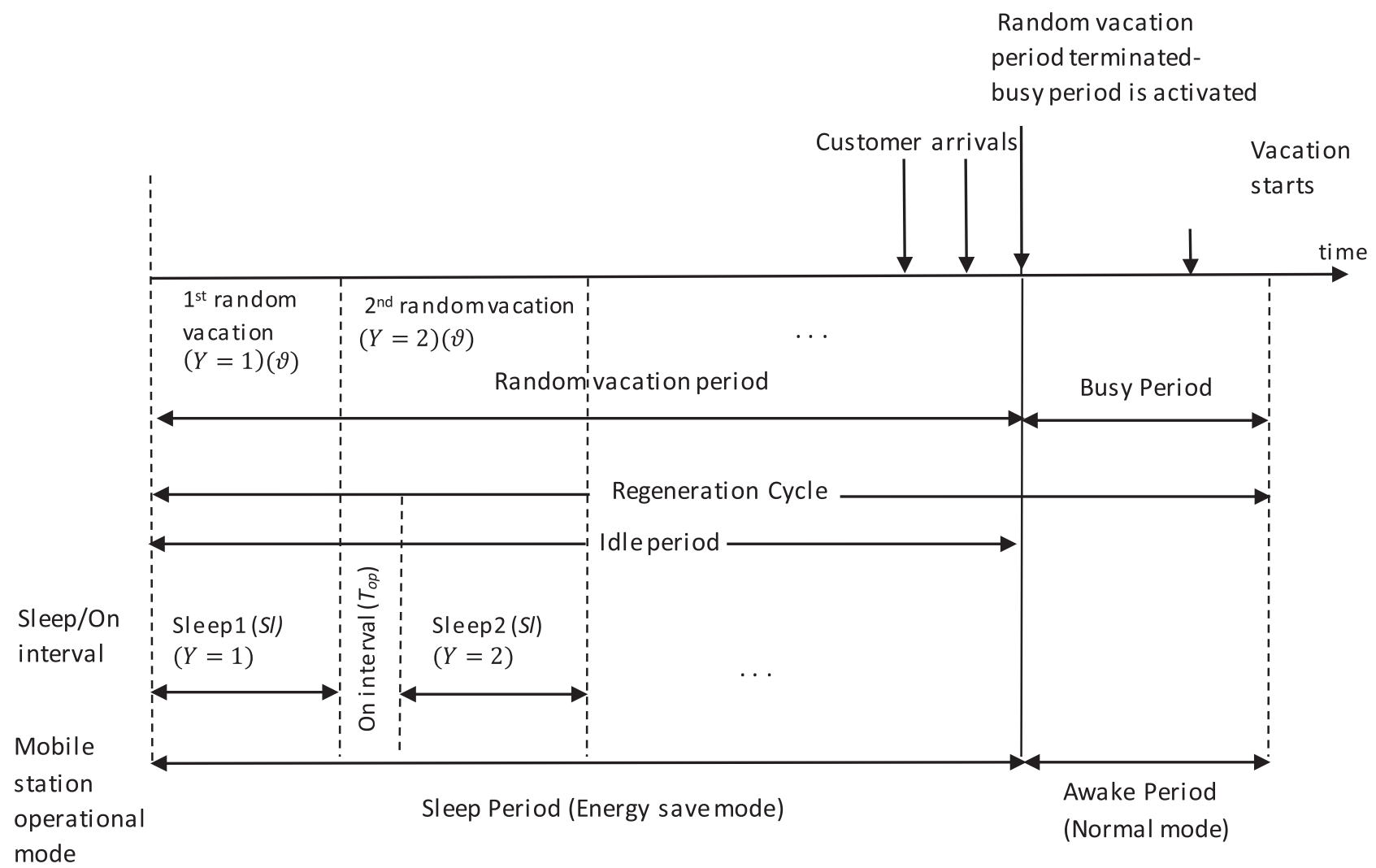

FiguRE 1. Mapping the $M / G / 1$ system with random vacation policy to the mobile station operational mode during type 1 cycle.

Here, we propose to study an $M / G / 1$ queueing system with a random vacation policy, where

Random vacation period (1st random vacation) = Sleep interval (Sleep 1) $(S l)$.

Random vacation period (Contains a maximum $Y$ number of random vacations) $=\mathrm{ON}$ interval $\left(T_{o p}\right)+$ Sleep interval (maximum $Y$ number of sleep intervals) $(S l), Y=2,3, \ldots$

Dormant period $=$ Warm-up period $\left(T_{w 1}\right)$.

Here the messages (customers) arrival process conforms to Poisson process and the delivering period of messages is generally distributed. The Poisson arrival process can be a practical consideration of voice traffic. Any new arrival during the random vacation period brings back the MS into the normal service period. These characteristics of the random vacation period in a random vacation policy (RVP) model can reduce holding time of an arriving message, which appears at a future stage of the sleep period.

\section{The MATHEMATiCAL MODEL}

In this section, we consider an $M / G / 1$ queue with random vacation policy, where the server takes a maximum number of $Y$ random vacations until the server finds minimum one customer in the queue. The detailed description of the model is stated below:

Assume that customers arrive to the queueing system according to a Poisson process with rate $\lambda$. The service time provided by a single server is an independently and identically distributed (i.i.d.) r.v $S$ with cumulative 


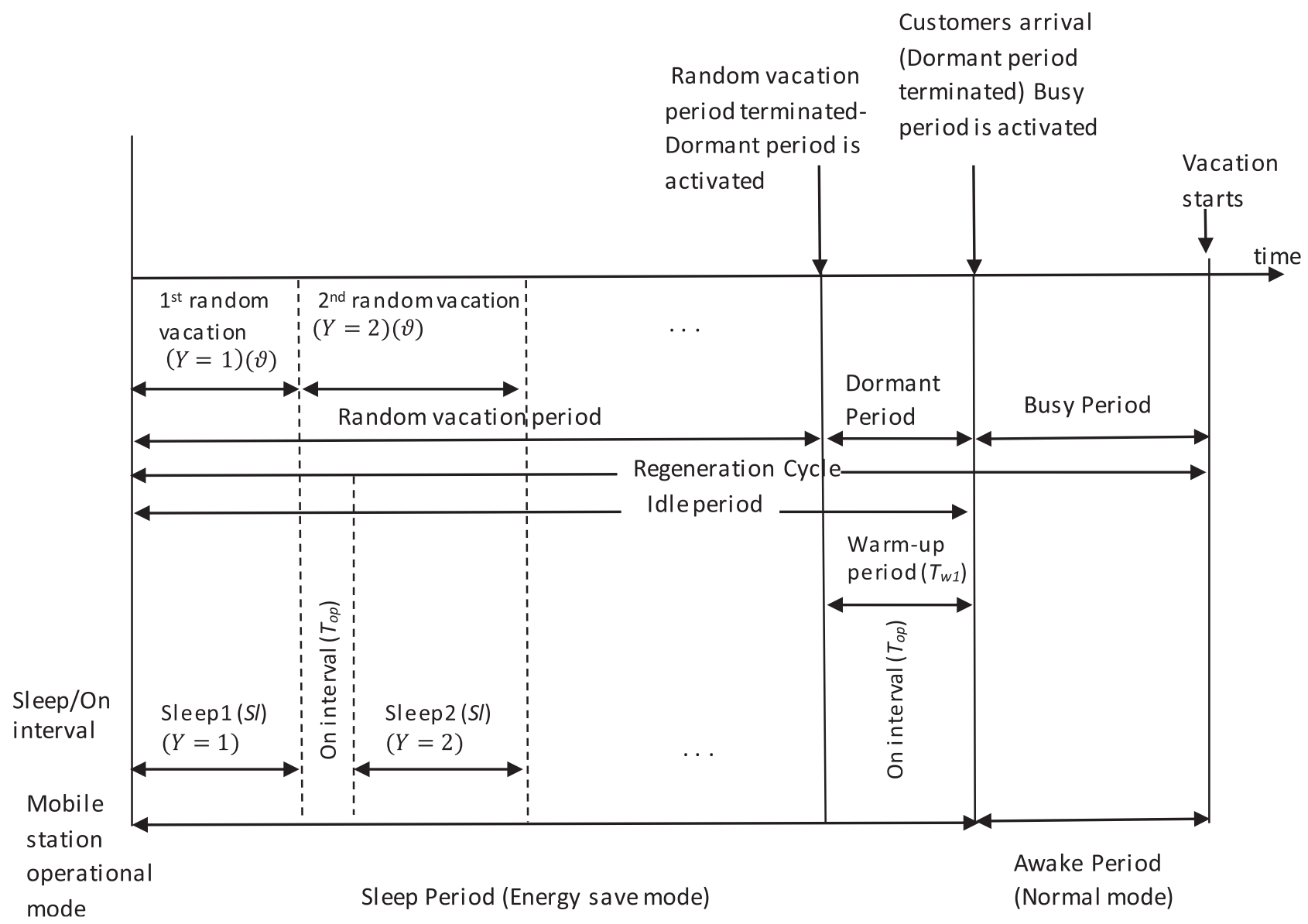

Figure 2. Mapping the $M / G / 1$ system with random vacation policy to the mobile station operational mode during type 2 cycle.

distribution function $(\mathrm{CDF}) F_{S}(x)$, probability density function $(\mathrm{PDF}) f_{S}(x)$, Laplace stieltjes transform (LST) $S^{*}(\theta)=E\left(\mathrm{e}^{-\theta S}\right)$ and finite moments $E\left(S^{r}\right) ; r=1,2 \ldots$ The customers are served singly by one server on a FCFS basis. Once the server finishes the service to all customers, i.e., at the end of each service period, the server enters into a vacation period of random duration $\vartheta$ (say). The duration of vacation period $\vartheta$ is i.i.d. r.v with CDF $F_{\vartheta}(x)$, the corresponding PDF $f_{\vartheta}(x)$, LST $\vartheta^{*}(\theta)=E\left(\mathrm{e}^{-\theta \vartheta}\right)$ and finite moments $E\left(\vartheta^{r}\right) ; r=1,2 \ldots$ On return from the initial vacation if the server finds no customers waiting in the queue it goes for the second vacation. This procedure will be continued until either the server finds minimum one customer at a vacation completion epoch or it has taken a maximum $Y$ number of random vacations (random vacation period). $Y$ is presumed to be a discrete r.v, such that

$$
P\{Y=j\}=y_{j} ; j=1,2, \ldots
$$

and $P_{Y}(z)=E\left(z^{Y}\right)$, its corresponding PGF. Moreover, the system remains idle during a random vacation period and a dormant period i.e., in case of Figure 1, random vacation period is an idle period and in case of Figure 2, random vacation period and dormant period together constitute an idle period.

Now, if we define $l_{j}$ and $\varrho_{j}$ as the probabilities that $j$ individual customers arrive during the service time $S$ and random vacation time $\vartheta$ respectively, then for $j=1,2, \ldots$, we have $l_{j}=\int_{0}^{\infty} \mathrm{e}^{-\lambda x}\left[\frac{(\lambda x)^{j}}{j !}\right] \mathrm{d} F_{S}(x)$ and 
$\varrho_{j}=\int_{0}^{\infty} \mathrm{e}^{-\lambda x}\left[\frac{(\lambda x)^{j}}{j !}\right] \mathrm{d} F_{\vartheta}(x)$. Therefore, the probability that $j$ individual customer arrive and are accepted during the random vacation time $\vartheta$ is $\frac{\varrho_{j}}{\left(1-\varrho_{0}\right)} ; j=1,2, \ldots$, where $\varrho_{0}=\vartheta^{*}(\lambda)$ is the probability that no customer arrives during a random vacation time $\vartheta$.

Further, it is presumed that arrival process, service time r.v, vacation time r.v and discrete r.v $Y$ are all mutually independent. It is essential to note that here we consider the concept of a warm-up period in an IEEE802.16e system, which refers to the time that is necessary for the server to waits for the first arrival after completing a random vacation period and this warm-up period is modeled as a dormant period in the underlying queueing system.

\section{Model analysis}

\subsection{Queue-size distribution at idle period completion epoch}

In this subsection, we derive the steady state queue size distribution at an idle period completion epoch.

First of all let us define the following events

$\gamma=$ No arrival occur during the random vacation period

and

$\varphi=$ Atleast one arrival occur during the random vacation period,

then, the probability of event $\gamma$ and $\varphi$ will be

$P(\gamma)=\sum_{n=1}^{\infty}\left(\vartheta^{*}(\lambda)\right)^{n} y_{n}=P_{Y}\left(\vartheta^{*}(\lambda)\right)$ and $P(\varphi)=1-P_{Y}\left(\vartheta^{*}(\lambda)\right)$.

Let $a_{n}$ denote the probability that $n$ customers arrived in the system at an idle period completion epoch. Therefore, by applying PASTA argument (see [29]), we can write

$$
a_{n}=\left[1-P_{Y}\left(\vartheta^{*}(\lambda)\right)\right] \frac{\varrho_{n}}{\left(1-\varrho_{0}\right)}+P_{Y}\left(\vartheta^{*}(\lambda)\right) \delta_{n, 1}, n=1,2, \ldots
$$

where

$$
\delta_{m, n}=\left\{\begin{array}{lll}
1, & \text { if } \quad & m=n \\
0, & \text { if } \quad m \neq n
\end{array}\right.
$$

denotes Kroneckers delta function. Then multiplying equation (4.1) by appropriate power of $z^{n}$ and taking summation over all possible values of $n$, we obtain

$$
\begin{aligned}
P_{A}(z)= & \sum_{n=1}^{\infty} a_{n} z^{n} \\
= & {\left[1-P_{Y}\left(\vartheta^{*}(\lambda)\right)\right] \sum_{n=1}^{\infty} \frac{\varrho_{n}}{\left(1-\varrho_{0}\right)} z^{n}+P_{Y}\left(\vartheta^{*}(\lambda)\right) \sum_{n=1}^{\infty} \delta_{n, 1} z^{n} } \\
= & \frac{\left(1-P_{Y}\left(\vartheta^{*}(\lambda)\right)\right.}{1-\vartheta^{*}(\lambda)}\left[\sum_{n=0}^{\infty} z^{n} \int_{0}^{\infty} \mathrm{e}^{-\lambda x} \frac{(\lambda x)^{n}}{n !} \mathrm{d} F_{\vartheta}(x)-z^{0} \int_{0}^{\infty} \mathrm{e}^{-\lambda x} \mathrm{~d} F_{\vartheta}(x)\right] \\
& +P_{Y}\left(\vartheta^{*}(\lambda)\right) \sum_{n=1}^{\infty} \delta_{n, 1} z^{n} \\
= & \left(1-P_{Y}\left(\vartheta^{*}(\lambda)\right)\left\{\frac{\left(P_{V}(z)-\vartheta^{*}(\lambda)\right)}{1-\vartheta^{*}(\lambda)}+\frac{P_{Y}\left(\vartheta^{*}(\lambda)\right) z}{1-P_{Y}\left(\vartheta^{*}(\lambda)\right.}\right\}\right.
\end{aligned}
$$

where $\vartheta^{*}(\lambda)=\int_{0}^{\infty} \mathrm{e}^{-\lambda x} \mathrm{~d} F_{\vartheta}(x)=\varrho_{0}$ and $P_{V}(z)=\sum_{n=0}^{\infty} z^{n} \int_{0}^{\infty} \mathrm{e}^{-\lambda x} \frac{(\lambda x)^{n}}{n !} \mathrm{d} F_{\vartheta}(x)=\vartheta^{*}(\lambda-\lambda z)$.

Clearly, $\left.\frac{\mathrm{d}}{\mathrm{d} z} P_{V}(z)\right|_{z=1}=\lambda E(\vartheta)$.

The mean and second moments are given by

$$
E(A)=\left.\frac{\mathrm{d}}{\mathrm{d} z} P_{A}(z)\right|_{z=1}=\left(1-P_{Y}\left(\vartheta^{*}(\lambda)\right)\left\{\frac{\lambda E(\vartheta)}{1-\vartheta^{*}(\lambda)}+\frac{P_{Y}\left(\vartheta^{*}(\lambda)\right)}{1-P_{Y}\left(\vartheta^{*}(\lambda)\right.}\right\}\right.
$$


and

$$
\begin{aligned}
E\left(A^{2}\right) & =\left.\frac{\mathrm{d}^{2}}{\mathrm{~d} z^{2}} P_{A}(z)\right|_{z=1}+\left.\frac{\mathrm{d}}{\mathrm{d} z} P_{A}(z)\right|_{z=1} \\
& =\left(1-P_{Y}\left(\vartheta^{*}(\lambda)\right)\left\{\frac{\lambda E(\vartheta)+\lambda^{2} E\left(\vartheta^{2}\right)}{1-\vartheta^{*}(\lambda)}+\frac{P_{Y}\left(\vartheta^{*}(\lambda)\right)}{1-P_{Y}\left(\vartheta^{*}(\lambda)\right.}\right\},\right.
\end{aligned}
$$

respectively. Note that the expression (4.3) represents the expected number of customers in the queue at the idle period completion epoch.

\subsection{Queue-size distribution at service completion epoch}

In this subsection, we derived the PGF of queue size distribution at service completion epoch. Let $t_{m}$ denotes the epoch at which $m-t h$ service completed. Then, the sequence $H_{m}=Z\left(t_{m}^{+}\right)$(where $Z\left(t_{m}\right)$ represents the number of customers to the system at time epoch $t_{m}$ ) construct a discrete time Markov chain (DTMC), which is a embedded Markov Chain in the underlying queueing system. We notice that $\left\{H_{m}, m \geq 0\right\}$ satisfies the following transition

$$
H_{m+1}=\left\{\begin{array}{l}
H_{m}-1+L_{m+1} \quad \text { while } H_{m}>0 \\
A_{m+1}+L_{m+1}-1 \text { while } H_{m}=0
\end{array},\right.
$$

where $L_{m}$ represents the number of arrivals occur during the $m$ th service time and $A_{m}$ represents the number of arrivals occur during the $m$ th idle period.

It is clear that $\left\{H_{m}, m=0,1, \ldots\right\}$ is an irreducible and aperiodic discrete time Markov chain. Therefore, the one- step transition probabilities of $H_{m}$ are given by

$$
P_{i, j}= \begin{cases}\sum_{c=1}^{j+1}\left[\left\{1-P_{Y}\left(\vartheta^{*}(\lambda)\right)\right\} \frac{\varrho_{c}}{\left(1-\varrho_{0}\right)}+P_{Y}\left(\vartheta^{*}(\lambda)\right) \delta_{c, 1}\right] l_{j-c+1} & \text { if } \quad i=0, j=0,1, \ldots \\ l_{j-i+1} & \text { if } \quad i \geq 1, j=i-1, i, \ldots \\ 0 & \text { if } \quad i \geq 1, j=0,1, \ldots, i-2 .\end{cases}
$$

Now, it is presumed that the Markov chain $\left\{H_{m}, m=0,1, \ldots\right\}$ is positive recurrent to ensure that the following limiting probabilities

$$
\pi_{j}=\lim _{m \rightarrow \infty} P\left[H_{m}=j\right], j=0,1, \ldots
$$

exist. Using (4.5), we can write the steady state equation as

$$
\pi_{j}=\pi_{o} \sum_{c=1}^{j+1}\left[\left\{1-P_{Y}\left(\vartheta^{*}(\lambda)\right)\right\} \frac{\varrho_{c}}{\left(1-\varrho_{0}\right)}+P_{Y}\left(\vartheta^{*}(\lambda)\right) \delta_{c, 1}\right] l_{j-c+1}+\sum_{c=1}^{j+1} \pi_{c} l_{j-c+1}, j=0,1, \ldots
$$

Now, we introduce the following generating functions for $|z| \leq 1$ :

$$
P_{L}(z)=\sum_{j=0}^{\infty} z^{j} l_{j}, \text { and } P_{\Pi}(z)=\sum_{j=0}^{\infty} z^{j} \pi_{j} .
$$

Clearly, $P_{L}(z)=\sum_{j=0}^{\infty} z^{j} \int_{0}^{\infty} \mathrm{e}^{-\lambda x} \frac{(\lambda x)^{j}}{j !} \mathrm{d} F_{S}(x)=S^{*}(\lambda-\lambda z)$ and $\left.\frac{\mathrm{d}}{\mathrm{d} z} P_{L}(z)\right|_{z=1}=\lambda E(S)$.

Now, multiplying both sides of equation (4.6) by $z^{j}$ and then taking summation over $j=0,1, \ldots$, we get

$$
P_{\Pi}(z)=\frac{\pi_{0}\left[1-\left\{\left(1-P_{Y}\left(\vartheta^{*}(\lambda)\right)\right)\left\{\frac{\left(P_{V}(z)-\vartheta^{*}(\lambda)\right)}{1-\vartheta^{*}(\lambda)}+\frac{P_{Y}\left(\vartheta^{*}(\lambda)\right) z}{1-P_{Y}\left(\vartheta^{*}(\lambda)\right)}\right\}\right\}\right] P_{L}(z)}{\left(P_{L}(z)-z\right)} .
$$

Now, applying the normalizing condition $P_{\Pi}(1)=1$, we obtain

$$
\pi_{0}=\frac{(1-\lambda E(S))\left(1-\vartheta^{*}(\lambda)\right)\left(1-P_{Y}\left(\vartheta^{*}(\lambda)\right)\right)^{-1}}{\left[\lambda E(\vartheta)+\frac{\left(1-\vartheta^{*}(\lambda)\right) P_{Y}\left(\vartheta^{*}(\lambda)\right)}{1-P_{Y}\left(\vartheta^{*}(\lambda)\right)}\right]} .
$$


Utilizing equations (4.8) in (4.7), we obtain

$$
P_{\Pi}(z)=\frac{(1-\lambda E(S))\left(1-\vartheta^{*}(\lambda)\right)\left[\frac{1}{\left(1-P_{Y}\left(\vartheta^{*}(\lambda)\right)\right)}-\left\{\frac{\left(P_{V}(z)-\vartheta^{*}(\lambda)\right)}{1-\vartheta^{*}(\lambda)}+\frac{P_{Y}\left(\vartheta^{*}(\lambda)\right) z}{1-P_{Y}\left(\vartheta^{*}(\lambda)\right)}\right\}\right] P_{L}(z)}{\left[\lambda E(\vartheta)+\frac{\left(1-\vartheta^{*}(\lambda)\right) P_{Y}\left(\vartheta^{*}(\lambda)\right)}{1-P_{Y}\left(\vartheta^{*}(\lambda)\right)}\right]\left(P_{L}(z)-z\right)},
$$

which is the PGF of queue size distribution at service completion epoch.

Let $E(\Pi)$ be the mean queue size at service completion epoch, then by differentiating equation (4.9) with regard to $\mathrm{z}$ and taking limit as $z \rightarrow 1$, we obtain

$$
E(\Pi)=\frac{2 \lambda E(S)+\lambda^{2}\left(E\left(S^{2}\right)-2(E(S))^{2}\right)}{2(1-\lambda E(S))}+\frac{\lambda^{2} E\left(\vartheta^{2}\right)}{2\left[\lambda E(\vartheta)+\frac{\left(1-\vartheta^{*}(\lambda)\right) P_{Y}\left(\vartheta^{*}(\lambda)\right)}{1-P_{Y}\left(\vartheta^{*}(\lambda)\right)}\right]} .
$$

Remark 4.1. It can be observed from equation (4.8) that $\lambda E(S)<1$, which is a necessary and sufficient condition for the stable solution of our model. Since $\lambda E(S)<1$, therefore the equation $P_{L}(z)-z=0$ never vanishes inside the region $|z| \leq 1$, which can be proved by using Rouche's theorem (see [25]).

\subsection{Steady state system size probabilities}

To obtain an explicit expression for steady state system size probabilities, we follow the method of Markov regenerative process, which gives much quicker result than the more popular method of supplementary variables techniques.

Let $h \in\{0,1, \ldots\}$ denotes the total number of customers in the system and $s \in\{0,1,2\}$ indicates whether the system is in random vacation period (which consists of maximum $Y$ number of random vacations) or dormant period or busy period at time $t$. Thus, the state of the system can be described by a 2-tuple or ordered pair $(h, s) \in\{0,1, \ldots\} \times\{0,1,2\} \equiv \Omega$. Consider the system state process $\{U(t), t \geq 0\}$, where $U(t)$ denotes the total number of customers in the system at time $t$. The process $\{U(t), t \geq 0\}$ is not a continuous time Markov chain (CTMC) since the sojourn time in each state is not exponentially distributed. Moreover, $U(t)$ is not even a semi-Markov process since between two completion epochs (i.e., between two sequential regeneration epochs) system state can change due to customers arrivals in random vacation period or dormant period or service completion. Define $\left\{\tau_{n}, n=0,1, \ldots\right\}$ as the sequence of epochs at which either service is terminated or random vacation is terminated or dormant period is terminated and let $\zeta_{n} \in \Omega$ be the state of the system at these epochs. Here, the elements of $\Omega$ are called regeneration epochs and they occur at service completion epoch, random vacation completion epoch, dormant period completion epoch. Hence, we can claim that $\left\{\zeta_{n}, n=0,1, \ldots\right\}$ is a DTMC with one-step transition probability matrix $K(\infty)$. By observing stochastic process $\{U(t), t \geq 0\}$ at these epochs, we see that the sequence $\left\{\zeta_{n}, \tau_{n}\right\}$ is an embedded Markov renewal sequence and $\{U(t), t \geq 0\}$ is a MRGP (see [15]).

We now proceed to establish the local kernel $E(t)$ and global kernel $K(t)$ of the MRGP. To simplify our analysis, we first draw the state transition diagram and it is shown in Figure 3. In Figure 3, we apply an oval symbol with ordered pair $(h, s)$ (as stated above) to show the state and apply a directed line to show a state transition. There are two types of state transitions as follows.

- Transition with a generally distributed sojourn time (corresponding to the service time and random vacation time) and is denoted by $F_{S}($.$) and F_{\vartheta}($.$) with dotted lines respectively.$

- Transition with exponentially distributed sojourn time (corresponding to inter-arrival time) with transition rate $\sigma_{i j}(h, n), 0 \leq i, j \leq 2, h=0,1, \ldots, n=0,1, \ldots$ and is represented by the solid lines. The possible transitions and corresponding rates are given in the following table: 


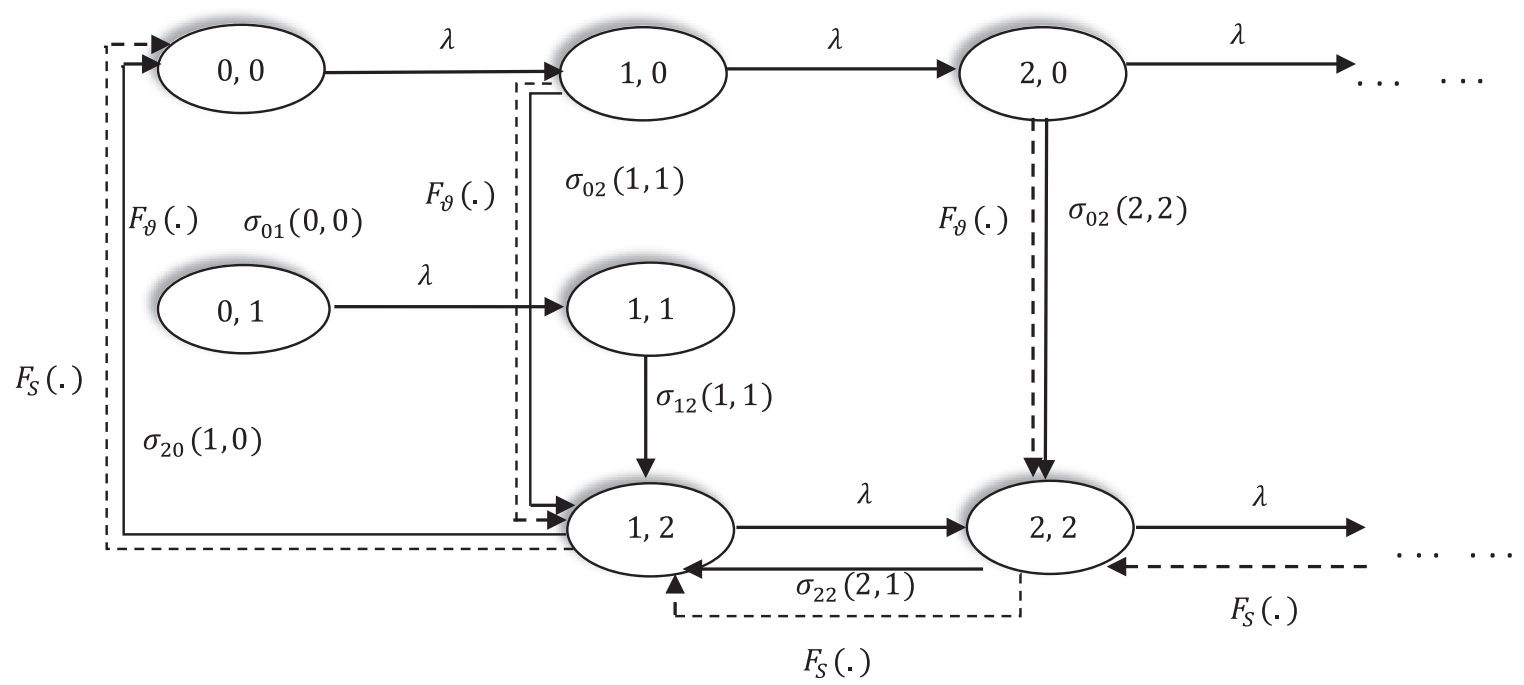

Figure 3. State transition diagram for $M / G / 1$ system with random vacation policy.

\begin{tabular}{llll}
\hline From & To & Transition rate & \\
\hline$(0,0)$ & $(0,1)$ & $\sigma_{01}(0,0)$ & for $h=n=1,2, \ldots$ \\
$(h, 0)$ & $(n, 2)$ & $\sigma_{02}(h, n)$ & \\
$(1,1)$ & $(1,2)$ & $\sigma_{12}(1,1)$ & \\
$(1,2)$ & $(0,0)$ & $\sigma_{20}(1,0)$ & for $h=2,3, \ldots, n=h-1$. \\
$(h, 2)$ & $(n, 2)$ & $\sigma_{22}(h, n)$ & \\
\hline
\end{tabular}

Now, to obtain the system state probabilities of MRGP, we find the closed form expressions for $K(t)$ and $E(t)$ as follows.

\subsubsection{Closed form expression for $K(t)$}

$K(t)$ is a global kernel matrix. The interpretation for the elements of the global kernel matrix $K(t)$ is as follows: $K_{(h, s) ;\left(n, s^{\prime}\right)}(t)$ denotes the probability that the system will be in state $\left(n, s^{\prime}\right), s^{\prime} \in\{0,1,2\}, n=0,1, \ldots$ at the time of the next regeneration epoch which occur on or before time $t$, given that it was in state $(h, s)$ just after the previous regeneration epoch. The evolution of MRGP between the Markov regeneration epochs can be explained through a CTMC infinitesimal generator matrix $Q$, as the only state transition that take place during this time are due to exponentially distributed interarrival times. The matrix $Q$ with the ordered pair $\{(0,0),(0,1),(1,0),(1,1),(1,2),(2,0),(2,1),(2,2), \ldots\}$ is as follows:

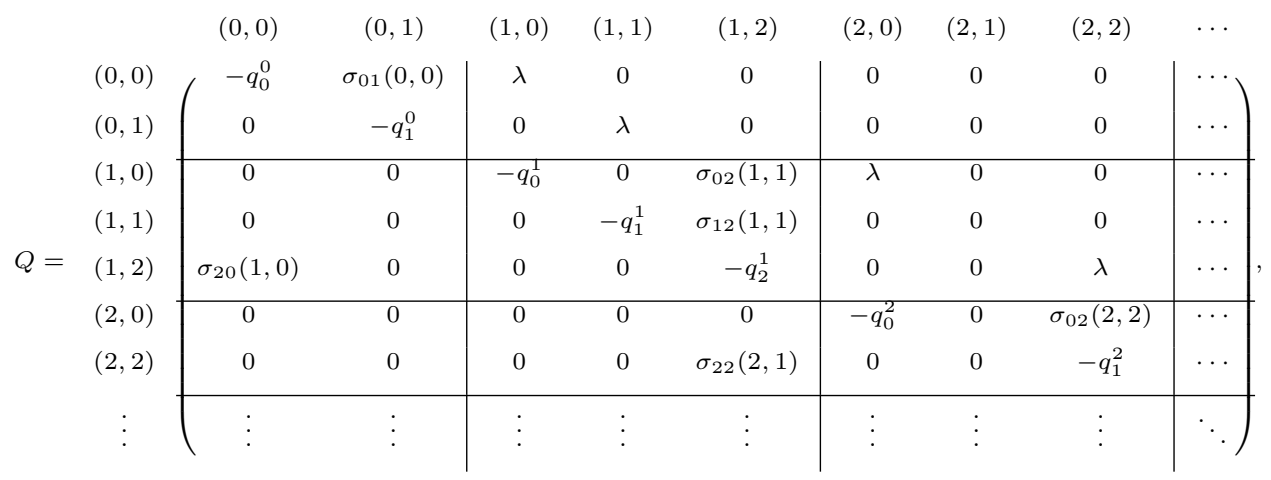


where $q_{0}^{0}=\left(\sigma_{01}(0,0)+\lambda\right), q_{1}^{0}=\lambda, q_{0}^{1}=\left(\sigma_{02}(1,1)+\lambda\right), q_{1}^{1}=\sigma_{12}(1,1), q_{2}^{1}=\left(\sigma_{20}(1,0)+\lambda\right), q_{0}^{2}=\left(\sigma_{02}(2,2)+\lambda\right)$, $q_{1}^{2}=\left(\sigma_{22}(2,1)+\lambda\right)$.

Let $N(t) \in\{0,1, \ldots\}$ be the total number of customers in the system during time interval $(0, t]$ and $D(t) \in$ $\{0,1,2\}$ be the state of the system. Then we define

$$
P_{i j}(h ; n, t)=P\{N(t)=n, D(t)=j \mid N(0)=h, D(0)=i\} ; n=0,1, \ldots, h=0,1, \ldots
$$

$P_{i j}(h ; n, t)$ is the probability that the subordinate CTMC will be in state $(n, j)$ during time $t$ given that it was in state $(h, i)$ initially. Let $\mathbf{P}(t)$ be the corresponding probability matrix. Define $\mathbf{P}(t)=$ $\left[P_{i 0}(h ; n, t), P_{i 1}(h ; n, t), P_{i 2}(h ; n, t)\right], i \in\{0,1,2\}, n=0,1, \ldots, h=0,1, \ldots .$. Suppose that $q_{j}^{\epsilon}<\infty, \epsilon=0,1,2, \ldots$, $j \in\{0,1,2\}$ but not all cases i.e., when $\epsilon=0, j \in\{0,1\}$, when $\epsilon=1, j=2$ and when $\epsilon=1,2, \ldots, j \in\{0,1\}$, therefore, $q_{j}^{\epsilon}$ represents $q_{0}^{0}, q_{1}^{0}, q_{0}^{1}, q_{1}^{1}, q_{2}^{1}, q_{0}^{2}, q_{1}^{2}, \ldots$, then the transition probabilities $P_{i j}(h ; n, t)$ are differentiable for all $t \geq 0$ and $i, j \in\{0,1,2\}, n=0,1, \ldots, h=0,1, \ldots$ and satisfy the following Kolmogorov forward equation

$$
P_{i j}^{\prime}(h ; n, t)=-q_{j}^{\epsilon} P_{i j}(h ; n, t)+\sum_{0 \leq k, j \leq 2} \sigma_{k j}(h ; n, t) P_{i k}(h ; n, t), P_{i i}(h ; h, 0)=1, i, j \in\{0,1,2\}, \epsilon=0,1,2, \ldots
$$

It can be written as

$$
\frac{\mathrm{d}}{\mathrm{d} t} \mathbf{P}(t)=\mathbf{P}(t) Q, P_{i i}(h ; h, 0)=1, i \in\{0,1,2\} .
$$

By solving equation (4.12), we obtain $P_{i j}(h ; n, t)$ and from the properties of transition probabilities, we get $\sigma_{i j}(h, n)=\lim _{t \rightarrow 0} \frac{P_{i j}(h ; n, t)}{t}<\infty, \forall 0 \leq i, j \leq 2, h=0,1, \ldots, n=0,1, \ldots$ Now, according to the definition of global kernel $K(t)$ in Kulkarni [15], we want to find

$$
K_{(h, s) ;\left(n, s^{\prime}\right)}(t)=P\left\{\zeta_{1}=\left(n, s^{\prime}\right), \tau_{1} \leq t \mid \zeta_{0}=(h, s)\right\}, 0 \leq s \leq 2, s^{\prime} \in\{0,1,2\},
$$

where $\tau_{1}$ is the first regeneration epoch.

Now, we define the elements of global kernel for each transition as follows.

Transition from random vacation period to dormant period. We presume the general transition of server random vacation. Server random vacation period (which consists of maximum $Y$ number of random vacations) $(i . e ., s=0)$ is enabled if and only if no customer is waiting in a queue, i.e., the system is empty. After completing a random vacation period if the server stays empty, it goes to a dormant period (i.e., $s=0, s^{\prime}=1$ ). Hence, we have

$$
K_{(0,0) ;(0,1)}(t)=\int_{0}^{t} P_{01}(0 ; 0, x) \mathrm{d} F_{\vartheta}(x) .
$$

Transition from random vacation period to service period. Here, we also consider the general transition of server random vacation. If any arrival occurs during the random vacation period, the server directly goes to an awake period or service period (i.e., $s=0, s^{\prime}=2$ ). Hence, we have

$$
K_{(h, 0) ;(n, 2)}(t)=\int_{0}^{t} P_{02}(h ; n, x) \mathrm{d} F_{\vartheta}(x), n=1,2, \ldots, h=0,1, \ldots
$$

Transition from dormant period to service period. Here, we presume the case of the server's dormant period, i.e., after completion of a random vacation period if the server finds no customer holding in a queue, it directly goes to a dormant period and waits for the first arrival. If any arrival occurs during the dormant period, it immediately goes to an awake period or service period (i.e., $s=1, s^{\prime}=2$ ). Thus, we have

$$
K_{(h, 1) ;(n, 2)}(t)=P_{12}(h ; n, t), n=1,2, \ldots, h=1,2, \ldots .
$$

Transition from service period to random vacation period. Here, we suppose that the customer service is the transition between two sequential regeneration epochs. When the server completes the service to all customers and no customer is waiting in the queue, i.e., the leaving customer is the final customer (i.e., $s=2, h=1)$, then the server goes to a random vacation period (i.e., $\left.s^{\prime}=0, n=0\right)$. Therefore, we have

$$
K_{(1,2) ;(0,0)}(t)=\int_{0}^{t} P_{20}(1 ; 0, x) \mathrm{d} F_{S}(x) .
$$


Transition from service period to service period. Here, we presume the general transition of the system's service period. Clearly, service period is available only when at least one customer is waiting in a queue. If the service termination of each customer does not empty the queue, the system stays in the service period (i.e., $s=2, s^{\prime}=2$ ). Hence, we have

$$
K_{(h, 2) ;(n, 2)}(t)=\int_{0}^{t} P_{22}(h ; n, x) \mathrm{d} F_{S}(x), n=1,2, \ldots, h=1,2, \ldots
$$

In summary, the global kernel $K_{(h, s) ;\left(n, s^{\prime}\right)}(t)$ of MRGP (see Eq. (4.13)) can be given as

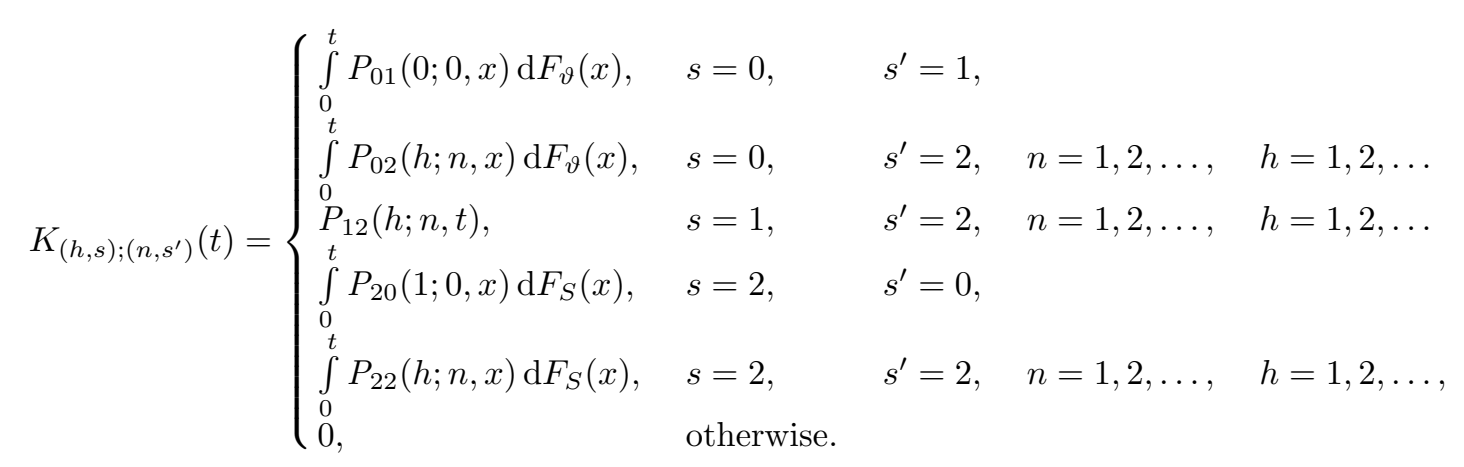

\subsubsection{Closed form expression for $E(t)$}

The local kernel matrix $E(t)$ describes the local behaviour of the process between two sequential regeneration epochs. The description for the elements of local kernel matrix $E(t)$ is as follows: $E_{(h, s) ;\left(n, s^{\prime}\right)}(t)$ denotes the probability that the system will be in state $\left(n, s^{\prime}\right)$ at time $t$ and the next regeneration epoch occurs after time $t$ given that the system was in state $(h, s)$ in the previous regeneration epoch. Hence, we can write the elements of $E(t)$ as

$$
E_{(h, s) ;\left(n, s^{\prime}\right)}(t)=P\left\{U(t)=\left(n, s^{\prime}\right), \tau_{1} \geq t \mid \zeta_{0}=(h, s)\right\}, 0 \leq s \leq 2, s^{\prime} \in\{0,1,2\} .
$$

Note that, $E_{(h, s) ;\left(n, s^{\prime}\right)}(t)=0$ for $s \in\{0,1,2\}$ and $s^{\prime} \in\{0,1\}$. Following the similar procedure in obtaining the global kernel $K(t)$, we find $E(t)$ as

$$
E_{(h, s) ;\left(n, s^{\prime}\right)}(t)=\left\{\begin{array}{lllll}
P_{02}(h ; n, t)\left(1-F_{\vartheta}(t)\right), & s=0, & s^{\prime}=2, & n=1,2, \ldots, & h=1,2, \ldots \\
P_{12}(h ; n, t), & s=1, & s^{\prime}=2, & n=1,2, \ldots, & h=1,2, \ldots \\
P_{22}(h ; n, t)\left(1-F_{S}(t)\right), & s=2, & s^{\prime}=2, & n=1,2, \ldots, & h=1,2, \ldots, \\
0, & \text { otherwise. } & &
\end{array}\right.
$$

\subsubsection{System state probabilities}

To obtain the system state probabilities, we define a new variable $\alpha_{s s^{\prime}}(h ; n)$, which represents the average amount of time the MRGP spends in state $s^{\prime}$ with $n$ customers between two sequential regeneration epochs, given that it was in state $s$ with $h$ customers after last regeneration:

$$
\alpha_{s s^{\prime}}(h ; n)=\int_{0}^{\infty} E_{(h, s) ;\left(n, s^{\prime}\right)}(t) \mathrm{d} t .
$$

It is easy to show that for $s=0, s^{\prime}=2$ and $n=1,2, \ldots, h=1,2, \ldots$

$$
\alpha_{02}(h ; n)=\left[1-P_{Y}\left(\vartheta^{*}(\lambda)\right)\right]\left\{1-\vartheta^{*}(\lambda)\right\}^{-1} \int_{0}^{\infty} \frac{\mathrm{e}^{-\lambda t}(\lambda t)^{n}}{n !}\left(1-F_{\vartheta}(t)\right) \mathrm{d} t
$$

For $s=1, s^{\prime}=2$ and $h=n=1,2, \ldots$

$$
\alpha_{12}(1 ; 1)=\frac{1}{\lambda}
$$


and for $s=2, s^{\prime}=2, n=1,2, \ldots$ and $h=1,2, \ldots$, we have

$$
\alpha_{22}(h ; n)=g_{n-h+1} ; n \geq \max (0, h-1),
$$

where $g_{n}=\int_{0}^{\infty} \mathrm{e}^{-\lambda t}\left[\frac{(\lambda t)^{n}}{n !}\right]\left(1-F_{S}(t)\right) \mathrm{d} t ; n=0,1, \ldots$

Since, the system state is basically based on the value of $h$ while $s \in\{0,1,2\}$, therefore, the system state probabilities are defined as

$$
\phi\left(n, s^{\prime}\right)=\frac{\sum_{h=0}^{\infty} \pi_{h} \alpha_{s s^{\prime}}(h ; n)}{\sum_{h=0}^{\infty} \pi_{h} \beta_{h}},
$$

where $\pi_{h}$ 's are the solution of $\pi=\pi K(\infty)$ and $\sum_{h=0}^{\infty} \pi_{h}=1$. Here, $\beta_{h}=\sum_{v \in\{0,1,2\}} \alpha_{v s^{\prime}}(h ; n) ; s^{\prime} \in\{0,1,2\}, n=$ $0,1, \ldots$ and $\sum_{h=0}^{\infty} \pi_{h} \beta_{h}=\frac{1}{\lambda}$.

Since, the arrival process is Poisson, therefore, by obeying Burke's theorem (see [4], pp. 187, 188) it is achieved that the system state probabilities $\left\{\phi\left(n, s^{\prime}\right) ;\left(n, s^{\prime}\right) \in \Omega\right\}$ are positive and exist under the similar condition of limiting probabilities $\left\{\pi_{n} ; n=0,1, \ldots\right\}$ of the Markov chain $\left\{\Xi_{m} ; m=0,1, \ldots\right\}$, i.e., if and only if $\lambda E(S)<1$.

Now, we consider the case of stationary system size distribution when the server state is in random vacation, i.e., $s^{\prime}=0$, a probability argument with (4.23) gives rise to

$$
\phi(n, 0)=\lambda \frac{\pi_{0}}{\left\{1-\vartheta^{*}(\lambda)\right\}}\left[1-P_{Y}\left(\vartheta^{*}(\lambda)\right)\right] \int_{0}^{\infty} \frac{\mathrm{e}^{-\lambda t}(\lambda t)^{n}}{n !}\left(1-F_{\vartheta}(t)\right) \mathrm{d} t ; n=0,1, \ldots
$$

Next, we consider the case of stationary system size distribution when the server state is in dormant period, i.e., $s^{\prime}=1$, a probability argument with (4.23) gives rise to

$$
\phi(1,1)=\pi_{0} P_{Y}\left(\vartheta^{*}(\lambda)\right)
$$

Lastly, we consider the case of stationary system size distribution when the server state is in busy period, i.e., $s^{\prime}=2$, a probability argument with (4.23) gives rise to

$$
\phi(n, 2)=\lambda\left[\pi_{o} \sum_{c=1}^{n+1}\left[\left\{1-P_{Y}\left(\vartheta^{*}(\lambda)\right)\right\} \frac{\varrho_{c}}{\left(1-\varrho_{0}\right)}+P_{Y}\left(\vartheta^{*}(\lambda)\right) \delta_{c, 1}\right] g_{n-c+1}+\sum_{c=1}^{n+1} \pi_{c} g_{n-c+1}\right], n=0,1, \ldots
$$

Now, we find the PGF of the above equations (4.24) and (4.26), which are given in the following theorem.

Theorem 4.2. Under stability condition $\lambda E(S)<1$, the PGF $P_{0}(z)$ of system size distribution when the server state is in random vacation period (i.e., $s^{\prime}=0$ ) is found as

$$
P_{0}(z)=\frac{(1-\lambda E(S))\left(1-P_{V}(z)\right)}{\left[\lambda E(\vartheta)+\frac{P_{Y}\left(\vartheta^{*}(\lambda)\right)\left(1-\vartheta^{*}(\lambda)\right)}{1-P_{Y}(\vartheta *(\lambda))}\right](1-z)}
$$

and the PGF $P_{2}(z)$ of system size distribution when the server is busy (i.e., $\left.s^{\prime}=2\right)$ is found as

$$
P_{2}(z)=\frac{(1-\lambda E(S))\left[\left(1-P_{V}(z)\right)+\frac{P_{Y}\left(\vartheta^{*}(\lambda)\right)\left(1-\vartheta^{*}(\lambda)\right)(1-z)}{1-P_{Y}\left(\vartheta^{*}(\lambda)\right)}\right]\left(1-P_{L}(z)\right)}{\left[\lambda E(\vartheta)+\frac{P_{Y}\left(\vartheta^{*}(\lambda)\right)\left(1-\vartheta^{*}(\lambda)\right)}{1-P_{Y}\left(\vartheta^{*}(\lambda)\right)}\right](1-z)\left(P_{L}(z)-z\right)} .
$$

Proof. Multiplying equations (4.24) and (4.26) by $z^{n}$ in both sides and summing over $n=0,1, \ldots$ and then utilizing the established result from renewal theory 


$$
\int_{0}^{\infty} \mathrm{e}^{-\theta x}(1-F(x)) \mathrm{d} x=\frac{1-f^{*}(\theta)}{\theta}
$$

where $F($.$) represents the CDF of an r.v and f^{*}($.$) its LST, we obtain the results (4.27) and (4.28)$ respectively.

Next, from the PGF of Theorem 4.2, we find the system state probabilities, where the server can be in random vacation period during a random vacation $(\mathrm{RV})$, in a dormant period $(D)$, in a busy period $(S)$ or in an idle period $(I)$, which are summarized in Corollary 4.3.

Corollary 4.3. For $\lambda E(S)<1$, the probability that the server is in random vacation period during a random vacation $P_{\mathrm{RV}}$, the probability that the server is in dormant period $P_{D}$, the probability that the server is in busy period $P_{S}$ and in idle period $P_{I}$ are obtained as

$$
\begin{aligned}
P_{\mathrm{RV}} & =\frac{(1-\lambda E(S)) \lambda E(\vartheta)}{\left[\lambda E(\vartheta)+\frac{\left(1-\vartheta^{*}(\lambda)\right) P_{Y}\left(\vartheta^{*}(\lambda)\right)}{1-P_{Y}\left(\vartheta^{*}(\lambda)\right)}\right]} . \\
P_{D} & =\frac{(1-\lambda E(S))\left(1-\vartheta^{*}(\lambda)\right) P_{Y}\left(\vartheta^{*}(\lambda)\right)}{\left(1-P_{Y}\left(\vartheta^{*}(\lambda)\right)\right)\left[\lambda E(\vartheta)+\frac{\left(1-\vartheta^{*}(\lambda)\right) P_{Y}\left(\vartheta^{*}(\lambda)\right)}{1-P_{Y}\left(\vartheta^{*}(\lambda)\right)} .\right.} . \\
P_{S} & =\lambda E(S) . \\
P_{I} & =1-\lambda E(S) .
\end{aligned}
$$

Proof. From equations (4.27) and (4.28) by taking limit as $z \rightarrow 1$ and after calculation, we obtain the results (4.29) and (4.31), i.e., $\lim _{z \rightarrow 1} P_{0}(z)=P_{\mathrm{RV}}, \phi(1,1)=P_{D}$ and $\lim _{z \rightarrow 1} P_{2}(z)=P_{S}$. By adding (4.29) and (4.30), equation (4.32) is obtained. Lastly, we find the PGF and mean of system size, which are given in Corollary 4.4.

Corollary 4.4. Let $N$ denotes the system size in steady state. Then, under the stability condition $\lambda E(S)<1$, the corresponding $P G F$ is given by

$$
\begin{aligned}
P_{N}(z)= & \frac{(1-\lambda E(S))(1-z) P_{L}(z)}{\left\{P_{L}(z)-z\right\}} \\
& \times \frac{\left(1-\vartheta^{*}(\lambda)\right)\left[\frac{1}{\left(1-P_{Y}\left(\vartheta^{*}(\lambda)\right)\right)}-\left\{\frac{\left(P_{V}(z)-\vartheta^{*}(\lambda)\right)}{1-\vartheta^{*}(\lambda)}+\frac{P_{Y}\left(\vartheta^{*}(\lambda)\right) z}{1-P_{Y}\left(\vartheta^{*}(\lambda)\right)}\right\}\right]}{\left[\lambda E(\vartheta)+\frac{\left(1-\vartheta^{*}(\lambda)\right) P_{Y}\left(\vartheta^{*}(\lambda)\right)}{1-P_{Y}\left(\vartheta^{*}(\lambda)\right)}\right](1-z)} .
\end{aligned}
$$

The mean of system size, $E(N)$ is given by

$$
E(N)=\frac{2 \lambda E(S)+\lambda^{2}\left(E\left(S^{2}\right)-2(E(S))^{2}\right)}{2(1-\lambda E(S))}+\frac{\lambda^{2} E\left(\vartheta^{2}\right)}{2\left[\lambda E(\vartheta)+\frac{\left(1-\vartheta^{*}(\lambda)\right) P_{Y}\left(\vartheta^{*}(\lambda)\right)}{1-P_{Y}\left(\vartheta^{*}(\lambda)\right)}\right]} .
$$

Proof. From equations (4.24) to (4.26), we get the probability of total system size in steady state as follows

$$
\xi_{n}=\phi(n, 0)+\phi(1,1)+\left(1-\delta_{n, 0}\right) \phi(n, 2) ; \text { for } n=0,1, \ldots,
$$

where $\xi_{n}=P(N=n)$. Then multiplying equation (4.35) by $z^{n}$ and taking summation over $n=0,1, \ldots$, we obtain

$$
P_{N}(z)=P_{0}(z)+\phi(1,1)+z P_{2}(z),
$$

which represents the connection between the 3 PGFs namely, $P_{N}(z), P_{0}(z)$ and $P_{2}(z)$ and probability $\phi(1,1)$. After simplifying equation (4.36), we obtain the formula (4.33), which is equal to the PGF of queue size distribution at service completion epoch. The result (4.34) follows directly from equation (4.33) on differentiation with respect to $z$ and assigning $z=1$. 
Remark 4.5. It is essential to note that the stochastic decomposition property (see [7]) can be exhibited for the underlying queueing system.

We can write the equation (4.33) as

$$
P_{N}(z)=P_{o}(z) P_{\Upsilon}(z)
$$

where $P_{o}(z)=\frac{\left(1-\lambda E(S)(1-z) P_{L}(z)\right.}{\left(P_{L}(z)-z\right)}$, the PGF of system size distribution of an ordinary $M / G / 1$ queue and

$$
P_{\Upsilon}(z)=\frac{\left(1-\vartheta^{*}(\lambda)\right)\left[\frac{1}{\left(1-P_{Y}\left(\vartheta^{*}(\lambda)\right)\right)}-\left\{\frac{\left(P_{V}(z)-\vartheta^{*}(\lambda)\right)}{1-\vartheta^{*}(\lambda)}+\frac{P_{Y}\left(\vartheta^{*}(\lambda)\right) z}{1-P_{Y}\left(\vartheta^{*}(\lambda)\right)}\right\}\right]}{\left[\lambda E(\vartheta)+\frac{\left(1-\vartheta^{*}(\lambda)\right) P_{Y}\left(\vartheta^{*}(\lambda)\right)}{1-P_{Y}\left(\vartheta^{*}(\lambda)\right)}\right](1-z)}
$$

is the PGF of additional system size distribution when the server is idle.

Thus, $P_{N}(z)=P_{o}(z) P_{\Upsilon}(z)$ confirms that the stochastic decomposition property of Fuhrmann and Cooper $[7]$ is also valid for the underlying queueing system under the stability condition.

\section{System Performance measures}

\subsection{Distribution of a number of customers served during the busy period}

Here, we derive the PGF and mean number of customers served during the busy period of this model under stability condition $\lambda E(S)<1$, which are given in the following theorem.

Theorem 5.1. Let $\psi$ denotes the number of customers served during the busy period. Then, under stability condition $\lambda E(S)<1$, the corresponding $P G F P_{\psi}(z)$ and mean $E(\psi)$ are determined as

$$
P_{\psi}(z)=\left(1-P_{Y}\left(\vartheta^{*}(\lambda)\right)\left\{\frac{\left(P_{V}(P(z))-\vartheta^{*}(\lambda)\right)}{1-\vartheta^{*}(\lambda)}+\frac{P_{Y}\left(\vartheta^{*}(\lambda)\right) P(z)}{1-P_{Y}\left(\vartheta^{*}(\lambda)\right.}\right\}\right.
$$

and

$$
E(\psi)=\frac{P_{Y}\left(\vartheta^{*}(\lambda)\right)}{1-\lambda E(S)}\left[1+\frac{\lambda E(\vartheta)\left(1-P_{Y}\left(\vartheta^{*}(\lambda)\right)\right)}{\left(1-\vartheta^{*}(\lambda)\right) P_{Y}\left(\vartheta^{*}(\lambda)\right)}\right]
$$

Proof. If $n$ customers are arrived in the system at the idle period completion epoch, then by utilizing the argument of Takagi [26] and equation (4.1), we have

$$
\begin{aligned}
P_{\psi}(z) & =\sum_{n=1}^{\infty} a_{n}(P(z))^{n} \\
& =\left(1-P_{Y}\left(\vartheta^{*}(\lambda)\right)\left\{\frac{\left(P_{V}(P(z))-\vartheta^{*}(\lambda)\right)}{1-\vartheta^{*}(\lambda)}+\frac{P_{Y}\left(\vartheta^{*}(\lambda)\right) P(z)}{1-P_{Y}\left(\vartheta^{*}(\lambda)\right.}\right\}\right.
\end{aligned}
$$

where $P(z)$ follows the following function equation under stability

$$
P(z)=z S^{*}(\lambda-\lambda P(z))
$$

Then differentiating equation (5.1) with regard to $z$ and assigning $z=1$, we obtain the result (5.2).

\subsection{Unfinished work}

In this subsection, our main objectives are to determine the LST of the distribution function of the unfinished work, the mean unfinished work and second moment of unfinished work. The following theorem summarizes the results: 
Theorem 5.2. Let $\varpi$ be the r.v representing the length of an unfinished work and $\varpi^{*}(\theta)$ be its corresponding $L S T$. For $\lambda E(S)<1$,

$$
\begin{aligned}
\varpi^{*}(\theta) & =\frac{\theta(1-\lambda E(S))\left[\left(1-P_{V}\left(S^{*}(\theta)\right)\right)+\frac{P_{Y}\left(\vartheta^{*}(\lambda)\right)\left(1-\vartheta^{*}(\lambda)\right)}{1-P_{Y}\left(\vartheta^{*}(\lambda)\right)}\left(1-S^{*}(\theta)\right)\right]}{\left[\lambda E(\vartheta)+\frac{P_{Y}\left(\vartheta^{*}(\lambda)\right)\left(1-\vartheta^{*}(\lambda)\right)}{1-P_{Y}\left(\vartheta^{*}(\lambda)\right)}\right]\left(\theta-\lambda+\lambda S^{*}(\theta)\right)\left(1-S^{*}(\theta)\right)} \\
L_{\varpi} & =E(\varpi)=\frac{\lambda^{2}(E(\vartheta))^{2} E\left(\vartheta_{r}\right)}{\left[\lambda E(\vartheta)+\frac{P_{Y}\left(\vartheta^{*}(\lambda)\right)\left(1-\vartheta^{*}(\lambda)\right)}{1-P_{Y}\left(\vartheta^{*}(\lambda)\right)}\right]}+\frac{\lambda E(S) E\left(S_{r}\right)}{(1-\lambda E(S))}
\end{aligned}
$$

and

$$
E\left(\varpi^{2}\right)=\frac{3 \lambda^{2} E(S) E\left(S^{2}\right) E\left(\vartheta^{2}\right)+\lambda^{3}(E(S))^{3} E\left(\vartheta^{3}\right)}{3 E\left(S^{2}\right)\left[\lambda E(\vartheta)+\frac{P_{Y}\left(\vartheta^{*}(\lambda)\right)\left(1-\vartheta^{*}(\lambda)\right)}{1-P_{Y}\left(\vartheta^{*}(\lambda)\right)}\right]}+\frac{E\left(S^{3}\right)}{E\left(S_{r}\right) 2 E(S)},
$$

where $E\left(S_{r}\right)=\frac{E\left(S^{2}\right)}{2 E(S)}$ and $E\left(\vartheta_{r}\right)=\frac{E\left(\vartheta^{2}\right)}{2 E(\vartheta)}$ represents the mean residual service time and vacation time respectively.

Proof. Let $\chi$ be the r.v describing the length of an unfinished work of an idle period and $\chi^{*}(\theta)$ be its LST, then by utilizing the standard argument of queueing theory (see [26]), we can write

$$
\begin{aligned}
\chi^{*}(\theta) & =P_{\Upsilon}\left(S^{*}(\theta)\right) \\
& =\frac{\left(1-\vartheta^{*}(\lambda)\right)\left[\frac{1}{\left(1-P_{Y}\left(\vartheta^{*}(\lambda)\right)\right)}-\left\{\frac{\left(P_{V}\left(S^{*}(\theta)\right)-\vartheta^{*}(\lambda)\right)}{1-\vartheta^{*}(\lambda)}+\frac{P_{Y}\left(\vartheta^{*}(\lambda)\right) S^{*}(\theta)}{1-P_{Y}\left(\vartheta^{*}(\lambda)\right)}\right\}\right.}{\left[\lambda E(\vartheta)+\frac{\left(1-\vartheta^{*}(\lambda)\right) P_{Y}\left(\vartheta^{*}(\lambda)\right)}{1-P_{Y}\left(\vartheta^{*}(\lambda)\right)}\right]\left(1-S^{*}(\theta)\right)} .
\end{aligned}
$$

Since, $\varpi^{*}(\theta)$ is the LST of the distribution function of the unfinished work, therefore, by utilizing the decomposition result of Leung [19], we can write

$$
\varpi^{*}(\theta)=\chi^{*}(\theta) \varpi_{M / G / 1}^{*}(\theta)
$$

where $\varpi_{M / G / 1}^{*}(\theta)=\frac{\theta(1-\lambda E(S))}{\theta-\lambda+\lambda S^{*}(\theta)}$ is the LST of the waiting time distribution of the first customer in the standard $M / G / 1$ queue. Thus, utilizing (5.7) in (5.8), the result (5.4) is obtained. By differentiating equation (5.4) with regard to $\theta$ and assigning $\theta=0$, formula (5.5) is obtained. Again, calculating the second differentiation of equation (5.4) with respect to $\theta$ and assigning $\theta=0$ and then adding the result with (5.5), after simplification we obtain the formula (5.6).

Remark 5.3. It is necessary to note that, the result (5.4) can be rearranged for the underlying queueing model and for that we need to define some other general descriptors which are listed below:

Let $T_{\mathrm{RV}}, T_{D}$ and $T_{I}$ denotes the length of a random vacation period, a dormant period and an idle period during a regeneration cycle respectively. Then the average residence times, say $E\left(T_{\mathrm{RV}}\right), E\left(T_{D}\right)$ and $E\left(T_{I}\right)$, of the server in a random vacation period, a dormant period and an idle period during a regeneration cycle are given by

$$
\begin{aligned}
E\left(T_{\mathrm{RV}}\right) & =\frac{P_{Y}\left(\vartheta^{*}(\lambda)\right) E(\vartheta)}{\left[\frac{P_{Y}\left(\vartheta^{*}(\lambda)\right)\left(1-\vartheta^{*}(\lambda)\right)}{1-P_{Y}(\vartheta *(\lambda))}\right]} . \\
E\left(T_{D}\right) & =\frac{P_{Y}\left(\vartheta^{*}(\lambda)\right)}{\lambda} . \\
E\left(T_{I}\right) & =E\left(T_{\mathrm{RV}}\right)+E\left(T_{D}\right)=\frac{\left[\lambda E(\vartheta) P_{Y}\left(\vartheta^{*}(\lambda)\right)+\frac{P_{Y}\left(\vartheta^{*}(\lambda)\right)^{2}\left(1-\vartheta^{*}(\lambda)\right)}{1-P_{Y}\left(\vartheta^{*}(\lambda)\right)}\right]}{\lambda\left[\frac{P_{Y}\left(\vartheta^{*}(\lambda)\right)\left(1-\vartheta^{*}(\lambda)\right)}{1-P_{Y}\left(\vartheta^{*}(\lambda)\right)}\right]} .
\end{aligned}
$$


Now, by utilizing the theory of regenerative process, we get the following conditional time average probabilities $\bar{P}_{\mathrm{RV}}$ and $\overline{P_{D}}$ as follows:

$$
\begin{aligned}
\bar{P}_{\mathrm{RV}} & =P(\text { server is in random vacation } / \text { server is idle }) \\
& =\frac{E\left(T_{\mathrm{RV}}\right)}{E\left(T_{I}\right)} \\
& =\frac{\lambda E(\vartheta)}{\left[\lambda E(\vartheta)+\frac{\left(1-\vartheta^{*}(\lambda)\right) P_{Y}\left(\vartheta^{*}(\lambda)\right)}{1-P_{Y}\left(\vartheta^{*}(\lambda)\right)}\right]}
\end{aligned}
$$

and

$$
\begin{aligned}
\bar{P}_{D} & =P(\text { server is in dormant period/server is idle }) \\
& =\frac{E\left(T_{D}\right)}{E\left(T_{I}\right)} \\
& =\frac{\left(1-\vartheta^{*}(\lambda)\right)}{\frac{\left(1-P_{Y}\left(\vartheta^{*}(\lambda)\right)\right)}{P_{Y}\left(\vartheta^{*}(\lambda)\right)}\left[\lambda E(\vartheta)+\frac{\left(1-\vartheta^{*}(\lambda)\right) P_{Y}\left(\vartheta^{*}(\lambda)\right)}{1-P_{Y}\left(\vartheta^{*}(\lambda)\right)}\right]} .
\end{aligned}
$$

By using the result of (5.12) and (5.13), we can rearrange the equation (5.4) as

$$
\varpi^{*}(\theta)=\frac{\bar{P}_{\mathrm{RV}} \theta(1-\lambda E(S))\left(1-P_{V}\left(S^{*}(\theta)\right)\right)+\bar{P}_{D}\left(\lambda E(\vartheta) \theta-\lambda^{2} E(\vartheta) \theta E(S)\right)\left(1-S^{*}(\theta)\right)}{\left(\theta-\lambda+\lambda S^{*}(\theta)\right)\left(1-S^{*}(\theta)\right) \lambda E(\vartheta)} .
$$

Now, by assigning $\bar{P}_{\mathrm{RV}}=1$ and $\bar{P}_{D}=0$ in equation (5.14), the result justifies the equation (3.7) of Takagi [26]. Similarly, by putting $\bar{P}_{\mathrm{RV}}=0$ and $\bar{P}_{D}=1$ in equation (5.14), the result verifies with Takagi [26].

\section{A particular CASE}

Here, we discuss momentarily a particular case of our model, which is consistent with the existing literature. Suppose we assume that the Probability mass function of the r.v $Y$ is

$$
y_{i}=p\left(1-\delta_{i, J}\right) \bar{p}^{i-1}+\delta_{i, J} \bar{p}^{J-1}, i=1,2, \ldots, J
$$

then the equations (4.2) and (4.9) yield

$$
P_{A}(z)=\frac{\left(1-\left(\bar{p} \vartheta^{*}(\lambda)\right)^{J}\right)\left(P_{V}(z)-\vartheta^{*}(\lambda)\right)}{1-\left(\bar{p} \vartheta^{*}(\lambda)\right)}+\left(\frac{p\left(\vartheta^{*}(\lambda)-\bar{p}^{J-1} \vartheta^{*}(\lambda)^{J}\right)}{1-\bar{p} \vartheta^{*}(\lambda)}+\bar{p}^{J-1} \vartheta^{*}(\lambda)^{J}\right) z
$$

and

$$
P_{\Pi}(z)=\frac{(1-\lambda E(S))\left(\frac{\left(1-\left(\bar{p} \vartheta^{*}(\lambda)\right)^{J}\right)\left(P_{V}(z)-1\right)}{\vartheta^{*}(\lambda)\left[\left(\bar{p} \vartheta^{*}(\lambda)\right)^{J-1}\left(1-\bar{p} \vartheta^{*}(\lambda)\right)+p\left(1-\left(\bar{p} \vartheta^{*}(\lambda)\right)^{J-1}\right)\right]}-1+z\right) P_{L}(z)}{E(S)\left(1+\frac{\lambda E(\vartheta)\left(1-\left(\bar{p} \vartheta^{*}(\lambda)\right)^{J}\right)}{\vartheta^{*}(\lambda)\left[\left(\bar{p} \vartheta^{*}(\lambda)\right)^{J-1}\left(1-\bar{p} \vartheta^{*}(\lambda)\right)+p\left(1-\left(\bar{p} \vartheta^{*}(\lambda)\right)^{J-1}\right)\right]}\right)\left(z-P_{L}(z)\right)} .
$$

Equations (6.2) and (6.3) represents the PGF of queue size distribution at an idle period completion epoch and service completion epoch of the $M / G / 1$ system having randomized vacation policy with at most $J$ vacations, where $J$ is a fixed number respectively. Note that for $J$ tends to $\infty$ and $p=0$, the above equations (6.2) and (6.3) are in accordance with the results obtained by Choudhury [3] for single unit arrival case. Also, we note that the equations (6.2) and (6.3) are consistent with Ke et al. [11] for single unit arrival case.

\section{Cost optimal policy}

In this section, we develop a long run average cost function for $M / G / 1$ queue in which we can compare different random vacation policies. To compare it, we build a homologous cost function that has been widely used in various practical works (see $[16,24]$ ). For this purpose, we need to find out an explicit expression for $E\left(S_{c}\right)$ defined as average length of the busy cycle which is given in the following theorem. 
Theorem 7.1. Under stability condition $\lambda E(S)<1$,

$$
E\left(S_{c}\right)=\frac{\lambda P_{Y}\left(\vartheta^{*}(\lambda)\right) E(\vartheta)+\frac{P_{Y}\left(\vartheta^{*}(\lambda)\right)^{2}\left(1-\vartheta^{*}(\lambda)\right)}{1-P_{Y}\left(\vartheta^{*}(\lambda)\right)}}{\lambda(1-\lambda E(S))\left[\frac{P_{Y}\left(\vartheta^{*}(\lambda)\right)\left(1-\vartheta^{*}(\lambda)\right)}{1-P_{Y}\left(\vartheta^{*}(\lambda)\right)}\right]} .
$$

Proof. Let $S_{c}$ denote the length of a busy cycle of a system which consists of a server's random vacation period $\left(T_{\mathrm{RV}}\right)$, dormant period $\left(T_{D}\right)$ and a busy period $\left(T_{S}\right)$ during a regeneration cycle, i.e., the cycle length $S_{c}$ can be written as $S_{c}=T_{\mathrm{RV}}+T_{D}+T_{S}$.

Now, we consider $T_{(1)}$ as the length of a busy period of the server started with a single customer. Then, under stability condition $\lambda E(S)<1$, the LST of $T_{(1)}$ in terms of Takacs functional equation (see [26]) is given by

$$
T_{(1)}^{*}(\theta)=S^{*}\left(\lambda+\theta-\lambda T_{(1)}^{*}(\theta)\right) .
$$

Differentiating the result of (7.2) with respect to $\theta$ and taking $\theta=0$, the average length of a busy period initiated by a single customer is found as

$$
E\left(T_{(1)}\right)=\frac{\lambda E(S)}{\lambda(1-\lambda E(S))}
$$

Now, we are going to find out the average residence time, say $E\left(T_{S}\right)$, during which the server is in busy period during a regeneration cycle. Clearly, if minimum one customer arrives during the random vacation period (with probability $1-P_{Y}\left(\vartheta^{*}(\lambda)\right)$ ), a busy period with one customer is started. In this case, the average residence time of the server in the busy period during a regeneration cycle (type 1 cycle) is

$$
\begin{aligned}
E\left(T_{S}^{1}\right) & =\frac{P_{Y}\left(\vartheta^{*}(\lambda)\right) E(\vartheta)}{\left[\frac{P_{Y}\left(\vartheta^{*}(\lambda)\right)\left(1-\vartheta^{*}(\lambda)\right)}{1-P_{Y}\left(\vartheta^{*}(\lambda)\right)} E\left(T_{(1)}\right)\right.} \\
& =\frac{P_{Y}\left(\vartheta^{*}(\lambda)\right) E(\vartheta)}{\left[\frac{P_{Y}\left(\vartheta^{*}(\lambda)\right)\left(1-\vartheta^{*}(\lambda)\right)}{1-P_{Y}\left(\vartheta^{*}(\lambda)\right)}\right]} \frac{\lambda E(S)}{\lambda(1-\lambda E(S))} .
\end{aligned}
$$

Now, we presume the case that the random vacation period has expired without any customer and the server enters into a dormant period. In this case, if minimum one customer arrives during the dormant period, a busy period is initiated. Then, the average residence time of the server in the busy period during a regeneration cycle (type 2 cycle) is

$$
E\left(T_{S}^{2}\right)=\frac{P_{Y}\left(\vartheta^{*}(\lambda)\right)}{\lambda} E\left(T_{(1)}\right)=\frac{P_{Y}\left(\vartheta^{*}(\lambda)\right) E(S)}{\lambda(1-\lambda E(S))} .
$$

Therefore, the total average residence time of the server in busy period during a regeneration cycle is given by

$$
E\left(T_{S}\right)=E\left(T_{S}^{1}\right)+E\left(T_{S}^{2}\right) .
$$

Finally, adding equations (5.9), (5.10) and (7.6), we obtain

$$
E\left(S_{c}\right)=E\left(T_{\mathrm{RV}}\right)+E\left(T_{D}\right)+E\left(T_{S}\right)=\frac{\lambda E(\vartheta) P_{Y}\left(\vartheta^{*}(\lambda)\right)+\frac{P_{Y}\left(\vartheta^{*}(\lambda)\right)^{2}\left(1-\vartheta^{*}(\lambda)\right)}{1-P_{Y}\left(\vartheta^{*}(\lambda)\right)}}{\lambda(1-\lambda E(S))\left[\frac{P_{Y}\left(\vartheta^{*}(\lambda)\right)\left(1-\vartheta^{*}(\lambda)\right)}{1-P_{Y}\left(\vartheta^{*}(\lambda)\right)}\right]},
$$

which represents the average length of a busy cycle of the system. 


\subsection{Average cost function}

Here, our purpose is to establish an optimum random vacation policy in order to minimize the expected cost function which is stated below. Let us define:

$C_{s u}=$ Setup cost per unit time for each busy cycle in the system. Thus, in long run the average setup cost is $\frac{C_{s u}}{E\left(S_{c}\right)}$.

$C_{h}=$ Holding cost per unit time for each customer available in the system. Therefore, in long run the average holding cost is $C_{h} E(\Pi)$

$C_{r v}=$ Cost due to the server is in random vacation. Therefore, in long run the average cost owing to server is in random vacation is $C_{r v} P_{\mathrm{RV}}$.

$C_{d}=$ Cost due to the server is in dormant. Thus, in long run the average cost owing to server is in dormant is $C_{d} P_{D}$.

$C_{S}=$ Per unit time cost for holding the server busy. Therefore, in long run the average cost owing to server busy is $C_{S} \lambda E(S)$.

Using the above cost parameter and renewal reward theory (see [22]), in long run the expected cost per unit time is given by

$$
T(\mathrm{RVP})=C_{s u} \frac{1}{E\left(S_{c}\right)}+C_{h} E(\Pi)+C_{r v} P_{\mathrm{RV}}+C_{d} P_{D}+C_{S} \lambda E(S) .
$$

We determine the optimal value of the control parameter by minimizing the average cost function obtained in equation (7.8). To find the optimum random vacation policy, i.e., where the cost is minimum, we have to select a locally optimal random vacation policy $\mathrm{RVP}^{*}$ so that in each selection it satisfies the following inequality

$$
T\left(\mathrm{RVP}^{1}\right) \geq T\left(\mathrm{RVP}^{2}\right) \geq T\left(\mathrm{RVP}^{*}\right),
$$

where $T\left(\mathrm{RVP}^{*}\right)$ depict the expected cost per unit time of locally optimal random vacation policy and $T\left(\mathrm{RVP}^{1}\right)$ and $T\left(\mathrm{RVP}^{2}\right)$ represents the expected cost per unit time of other two random vacation policies with different probability distributions of the discrete r.v $Y$. The above inequality holds because locally optimal random vacation policy $\left(\mathrm{RVP}^{*}\right)$ will always give the minimum expected cost per unit time and hence, the expected cost per unit time of locally optimal random vacation policy $\left(T\left(\mathrm{RVP}^{*}\right)\right)$ is less than or equal to the other two policies. The relation between the other two policies $T\left(\mathrm{RVP}^{1}\right)$ and $T\left(\mathrm{RVP}^{2}\right)$ is first policy $T\left(\mathrm{RVP}^{1}\right)$ will be greater than or equal to the other one $T\left(\mathrm{RVP}^{2}\right)$.

In order to show the applicability of cost optimal policy in our problem, we present six numerical examples for three different service time distributions and different cost parameters. For the purpose of numerical examples, We presume that the vacation time follows an exponential distribution with $\operatorname{PDF} f_{\vartheta}(x)=\nu \mathrm{e}^{-\nu x}, x>0$.

\section{NUMERICAL ANALYSIS}

In this section, we present the numerical results of the impacts of system parameters on the previously obtained key performance measures in the form of graphs. In the previous sections, we have obtained the probability $P_{\mathrm{RV}}$ of a server is in random vacation period during a random vacation, the probability $P_{D}$ of a server is in dormant period, mean number $E(\psi)$ of customers served during the busy period and mean unfinished work $L_{\varpi}$.

For the purpose of numerical analysis, we presume that the service time conforms to

- 2-stage Erlang distribution (denoted by 2-stage Erlang) with parameter $\mu$, then we have the PDF $f_{S}(x)=$ $\mu^{2} x \mathrm{e}^{-\mu x}, x>0, \operatorname{LST} S^{*}(\theta)=\frac{2 \mu}{(2 \mu+\theta)^{2}}$, mean $E(S)=\frac{2}{\mu}$ and second moment $E\left(S^{2}\right)=\frac{2(2+1)}{\mu^{2}}$ respectively.

- Exponential distribution (denoted by Exp) with parameter $\mu$, then we have the PDF $f_{S}(x)=\mu \mathrm{e}^{-\mu x}, x>0$, LST $S^{*}(\theta)=\frac{\mu}{\mu+\theta}$, mean $E(S)=\frac{1}{\mu}$ and second moment $E\left(S^{2}\right)=\frac{2}{\mu^{2}}$ respectively.

- Hyper-Exponential distribution (denoted by Hyp-Exp) with parameter $\mu$, then we have the PDF $f_{S}(x)=$ $0.5 \mu \mathrm{e}^{-\mu x}+0.5 \mu^{2} \mathrm{e}^{-\mu^{2} x}, x>0, \operatorname{LST} S^{*}(\theta)=\frac{0.5 \mu}{\mu+\theta}+\frac{0.5 \mu^{2}}{\mu^{2}+\theta}$, mean $E(S)=\frac{0.5(\mu+1)}{\mu^{2}}$ and second moment $E\left(S^{2}\right)=\frac{\mu+1}{\mu^{2}}$ respectively.

We also consider that the maximum number of vacations $Y$ follows 


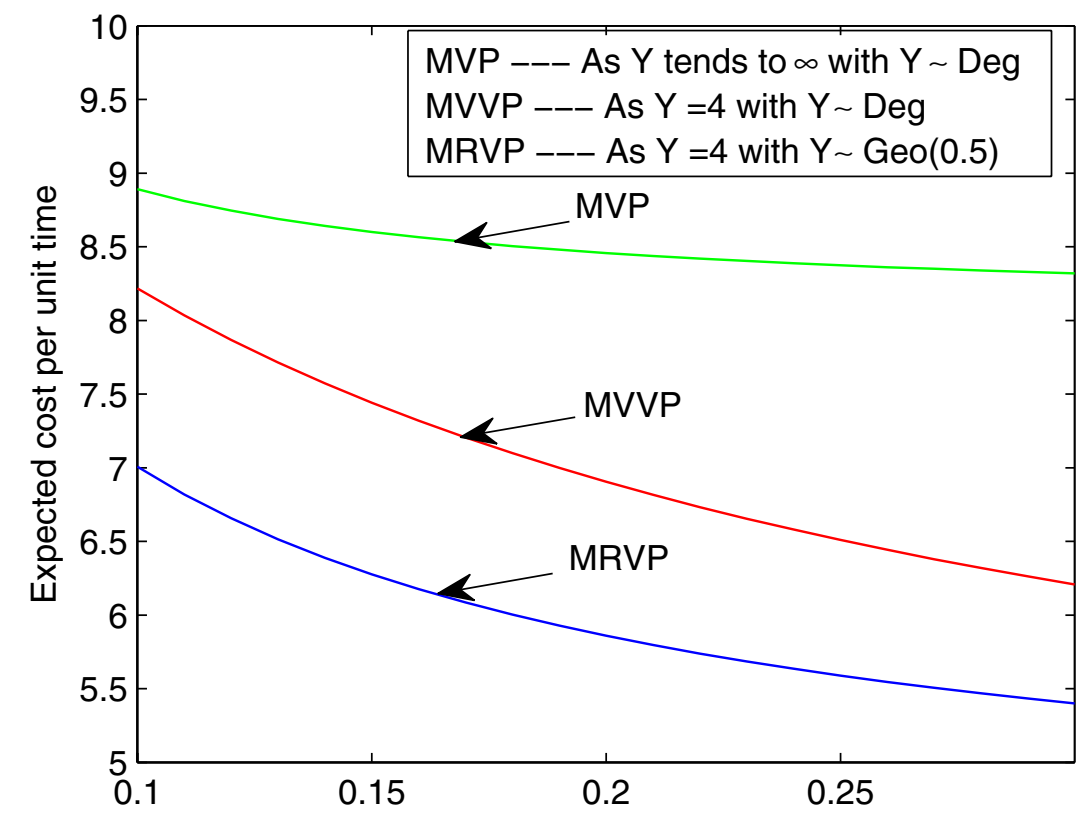

$v$, when $\lambda=0.05, \mu=8, C_{s u}=10, c_{h}=2, c_{r v}=7, c_{d}=3, c_{S}=50, S \sim 2-$ stage Erlang

FiguRE 4. Impact of $\nu$ on expected cost per unit time.

- Degenerate distribution (denoted by Deg) at point $j$, then we have the PMF $P\{Y=j\}=1$, PGF $P_{Y}(z)=$ $\frac{1}{1-z}$, mean $E(Y)=j$ and second moment $E\left(Y^{2}\right)=j^{2}$ respectively.

- Geometric distribution (denoted by Geo) with parameter $p$, then we have the $\operatorname{PMF} P\{Y=j\}=p(1-$ $p)^{j-1} ; j=1,2, \ldots, 0<p<1, \operatorname{PGF} P_{Y}(z)=\frac{p z}{1-(1-p) z}$, mean $E(Y)=\frac{1}{p}$ and second moment $E\left(Y^{2}\right)=\frac{2-p}{p^{2}}$ respectively.

Considering the cost parameters as $C_{s u}=10, C_{h}=2, C_{r v}=7, C_{d}=3, C_{S}=50$, model parameter as $\lambda=0.05$ and $\mu=8$, we display the impact of vacation rate $\nu$ on expected cost per unit time (7.8) for three vacation policies as well as for three service time distributions in Figures 4-6. Figures 7-9 also show the effect of $\nu$ on expected cost per unit time (7.8) for three vacation policies as well as for three service time distributions but here $C_{s u}=300$. Other parameters are same as in Figures $4-6$.

In Figures 4-6 as well as in Figures 7-9, we compare the expected cost per unit time of three vacation policies: multiple vacation policy (MVP), multiple variant vacation policy (MVVP) and multiple randomized vacation policy (MRVP) for three service time distributions namely, 2-stage Erlang, exponential and hyper-exponential. MVP is a policy where the server takes multiple numbers of consecutive vacations until it finds one customer waiting in a queue at a vacation completion epoch. MVVP is a policy where the server takes a fixed number of multiple vacations until it finds at least one customer waiting in a queue at a vacation completion epoch. If no customer is found after completing a fixed number of multiple vacations, the server stays dormant in the system and waits for the first arrival. MRVP is described as follows: After the system becomes empty, the server immediately goes for a vacation. If there is at least one customer waiting in the queue upon returning from a vacation, the server is immediately activated for service. Otherwise, if no customer is waiting in a queue at a vacation completion epoch, the server either remains idle in the system with probability $p$ or leaves for another vacation with probability $1-p$. This pattern continues until a fixed number of multiple vacations are completed. If the system is still empty after completing a fixed number of multiple vacations, it stays dormant in the system and waits for the first arrival. The effect of $\nu$ on expected cost per unit time are also shown in 


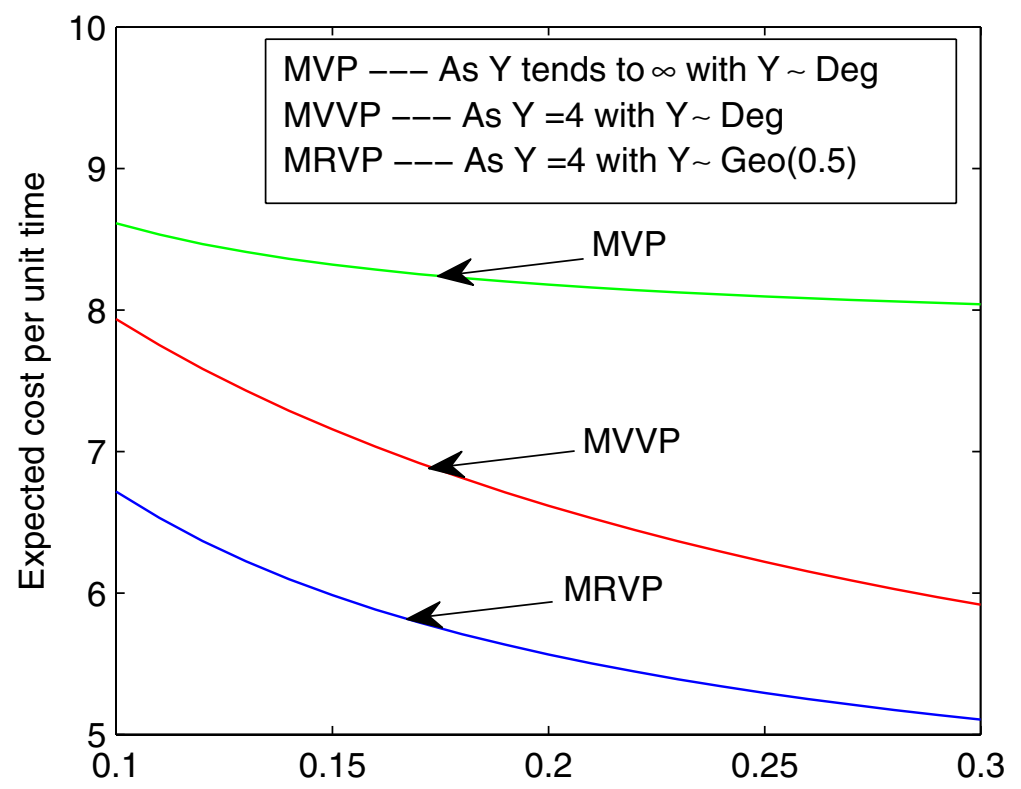

$v$, when $\lambda=0.05, \mu=8, C_{s u}=10, c_{h}=2, c_{r v}=7, c_{d}=3, c_{S}=50, S \sim \operatorname{Exp}$

FiguRE 5. Impact of $\nu$ on expected cost per unit time.

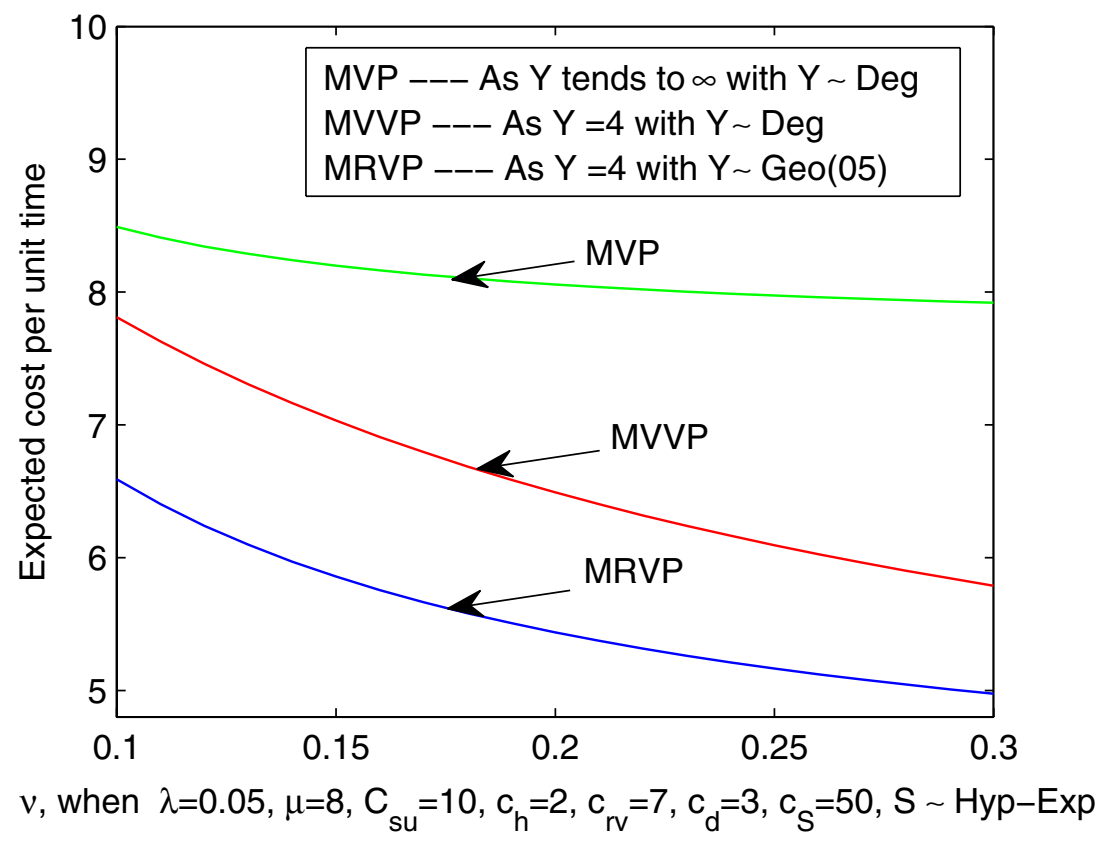

Figure 6 . Impact of $\nu$ on expected cost per unit time. 


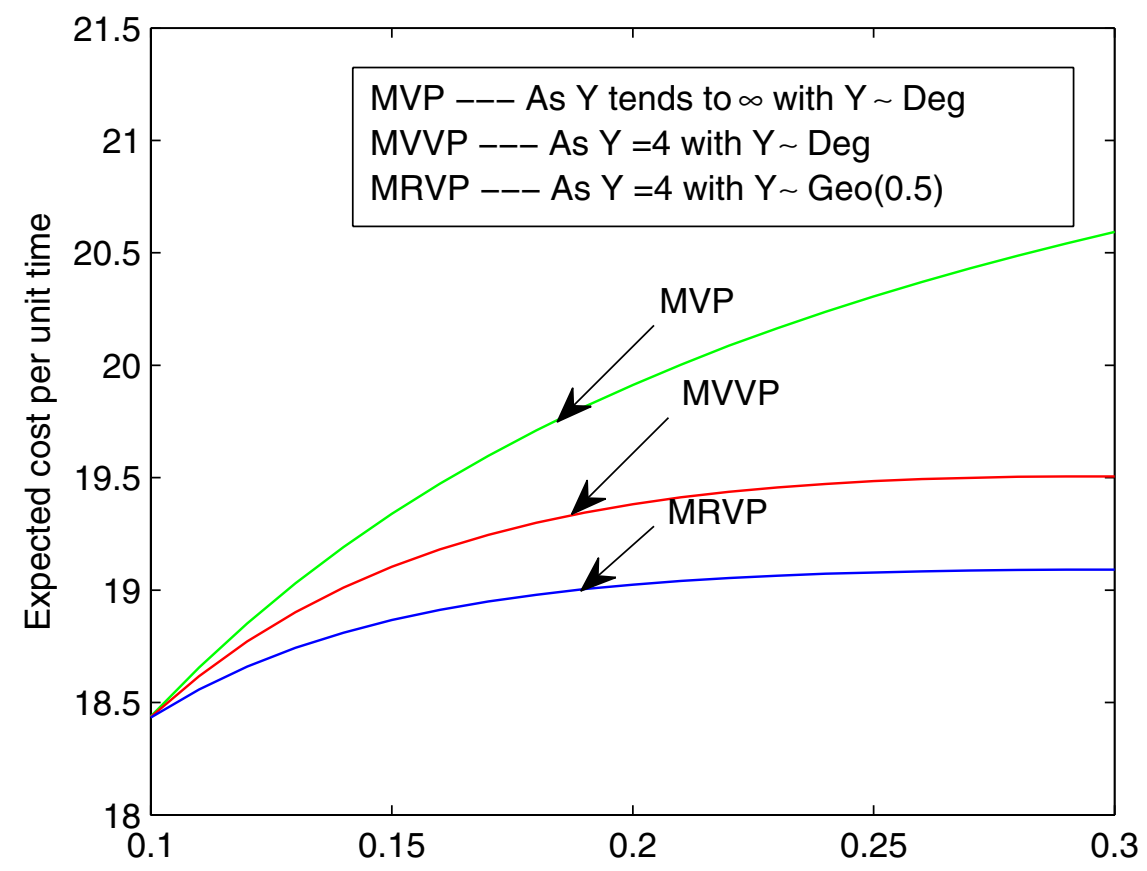

$v$, when $\lambda=0.05, \mu=8, C_{s u}=300, c_{h}=2, c_{r v}=7, c_{d}=3, c_{S}=50, S \sim 2$-stage Erlang

FiguRE 7 . Impact of $\nu$ on expected cost per unit time.

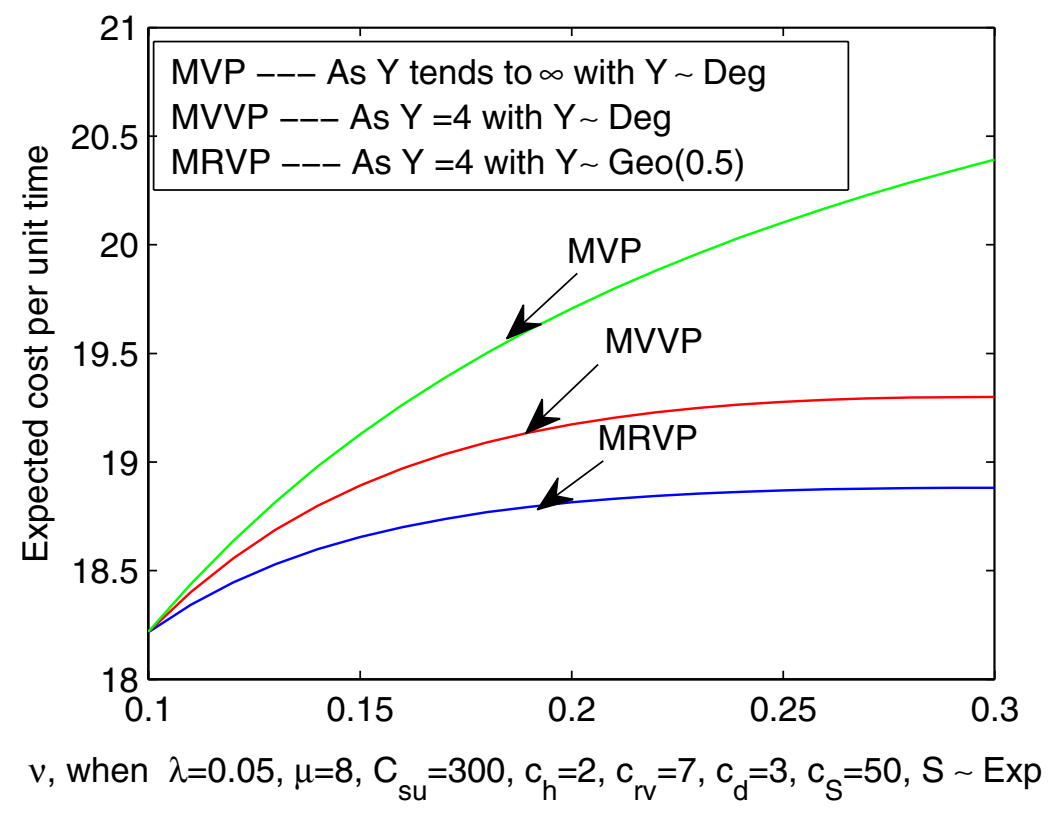

FiguRE 8. Impact of $\nu$ on expected cost per unit time. 


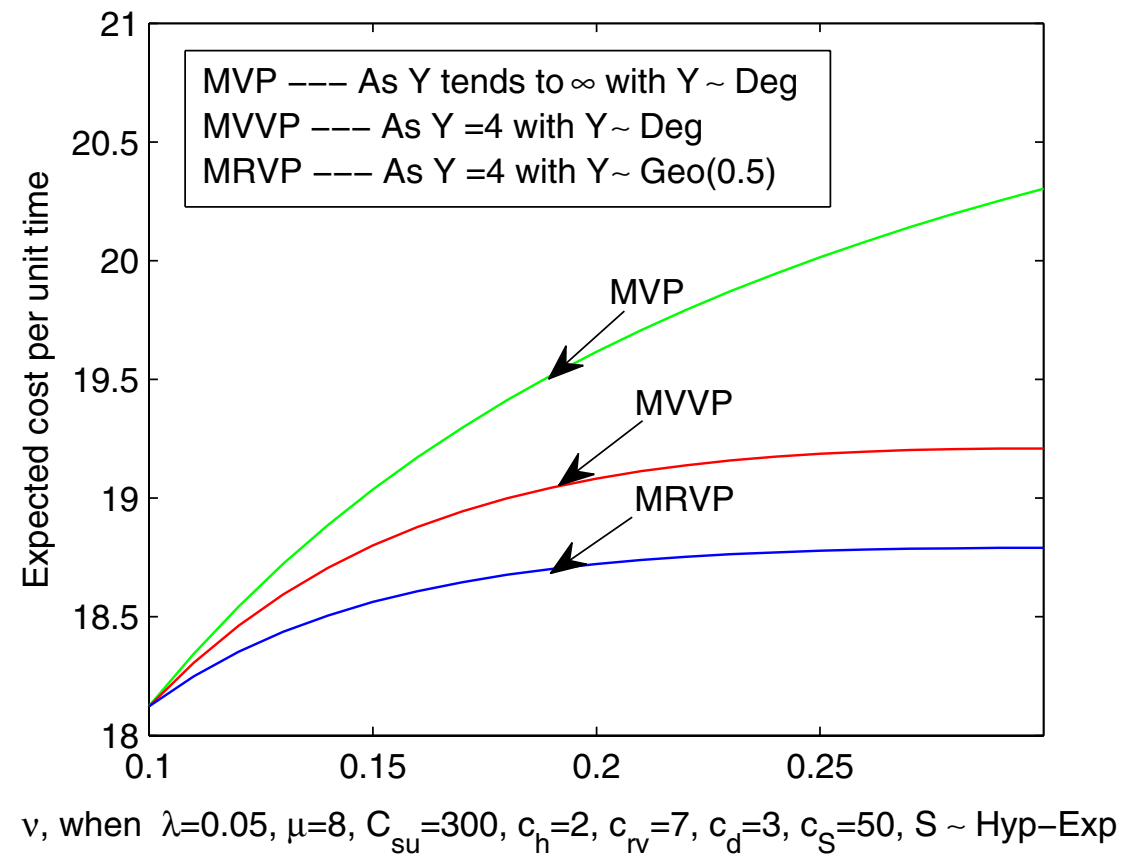

Figure 9. Impact of $\nu$ on expected cost per unit time.

Figures 4-6, when $C_{s u}=10$ and in Figures 7-9, when $C_{s u}=300$. We summarize some findings:

(1) When $Y$ tends to $\infty$ and $Y$ follows a degenerate distribution and $C_{s u}=10$, in Figures 4-6, we can see that expected cost decreases as the vacation rate $\nu$ increases whereas for $C_{s u}=300$, in Figures 7-9, we notice that as the vacation rate $\nu$ increases expected cost also increases before attains stability, this is because the model becomes an $M / G / 1$ system with multiple vacation policy.

(2) If $Y=4$, i.e., a finite value and $Y$ follows a degenerate distribution, the model becomes the $M / G / 1$ queue with multiple variant vacation policy and for $C_{s u}=10$, in Figures $4-6$, we see that the expected cost per unit time decreases as $\nu$ increases whereas for $C_{s u}=300$, in Figures 7-9, we observe that as $\nu$ increases expected cost also increases before it reaches stability.

(3) When $Y=4$ (a finite value) and $Y$ follows a geometric distribution with parameter $p=0.5$ and $C_{s u}=10$, in Figures 4-6, we can find that expected cost decreases as the values of $\nu$ increases whereas for $C_{s u}=300$, in Figures 7-9, we observe that as $\nu$ increases expected cost also increases, this is because that the model becomes an $M / G / 1$ system with randomized vacation policy.

It is clear that when $C_{s u}=10$ i.e., comparatively small, expected cost will be decreases as the values of $\nu$ increases, on the other hand, when $C_{s u}=300$ i.e., comparatively large, expected cost will be increases as the values of $\nu$ increases.

By comparing expected cost per unit time in terms of three vacation policies yields, MVP $>$ MVVP $>$ MRVP, i.e., MRVP is better than the two other policies. We also observe that the expected cost per unit time is larger for 2-stage Erlang service time distribution as compared to exponential and hyper-exponential distribution.

Therefore, if we want to minimize the expected cost per unit time, we can consider multiple randomized vacation policy as a optimal policy, which shows minimum expected cost per unit time compared to the expected cost of other two policies.

From a practical point of view, some other useful measures are the probability $P_{\mathrm{RV}}$ of a server is in random vacation period during a random vacation, the Probability $P_{D}$ of a server is in dormant period, mean number $E(\psi)$ of customers served during the busy period and mean unfinished work $L_{\varpi}$ respectively. 


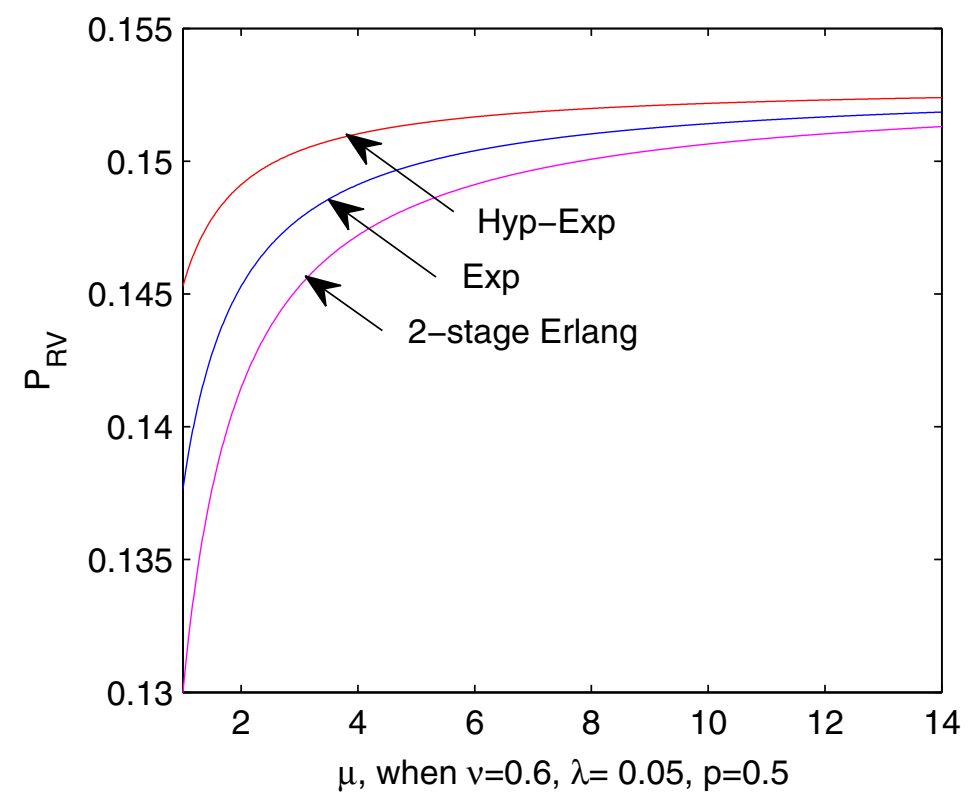

Figure 10. Impact of $\mu$ on $P_{\mathrm{RV}}$ for different service time distributions.

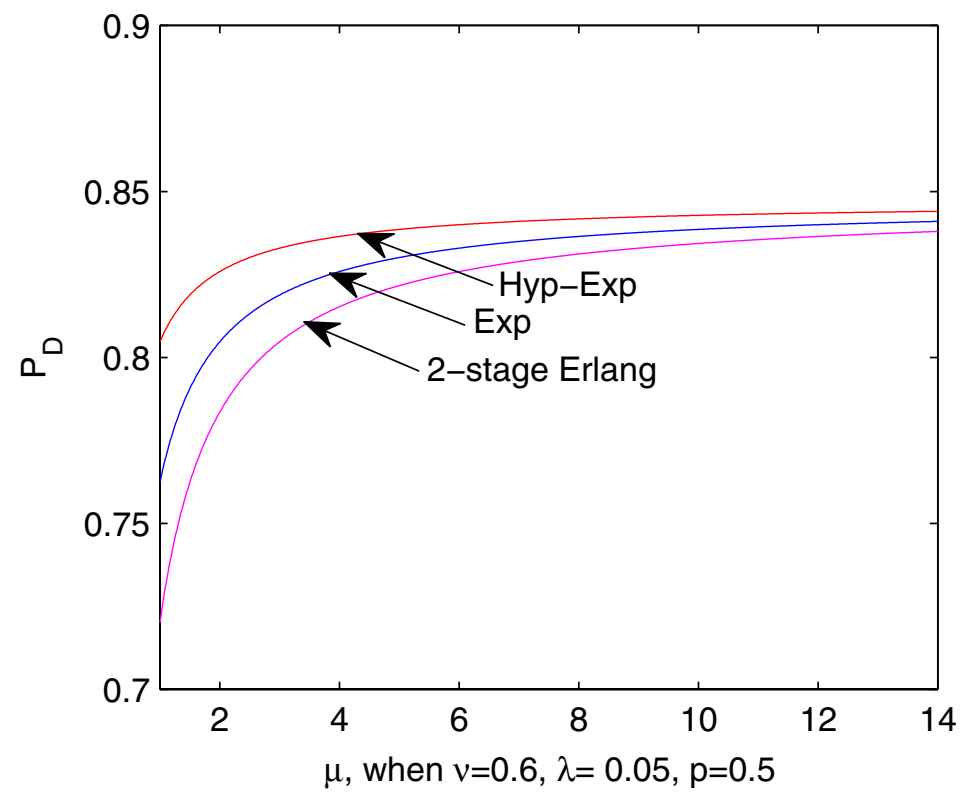

Figure 11. Impact of $\mu$ on $P_{D}$ for different service time distributions.

Now, Considering the vacation time as exponential distribution, while $\mathrm{Y}$ follows a geometric distribution and parametric values as $\lambda=0.05, \nu=0.6$ and $p=0.5$, we display the effect of service rate $\mu$ on $P_{\mathrm{RV}}, P_{D}, E(\psi)$ and $L_{\varpi}$ for three service time distributions namely, 2-stage Erlang, exponential and hyper-exponential in Figures 10-13.

While the other model parameters are fixed, Figures 10 and 11 show that the system state probability $P_{\mathrm{RV}}$ and $P_{D}$ increases as service rate $\mu$ increases before it arrives stability. This is because as the values of service 


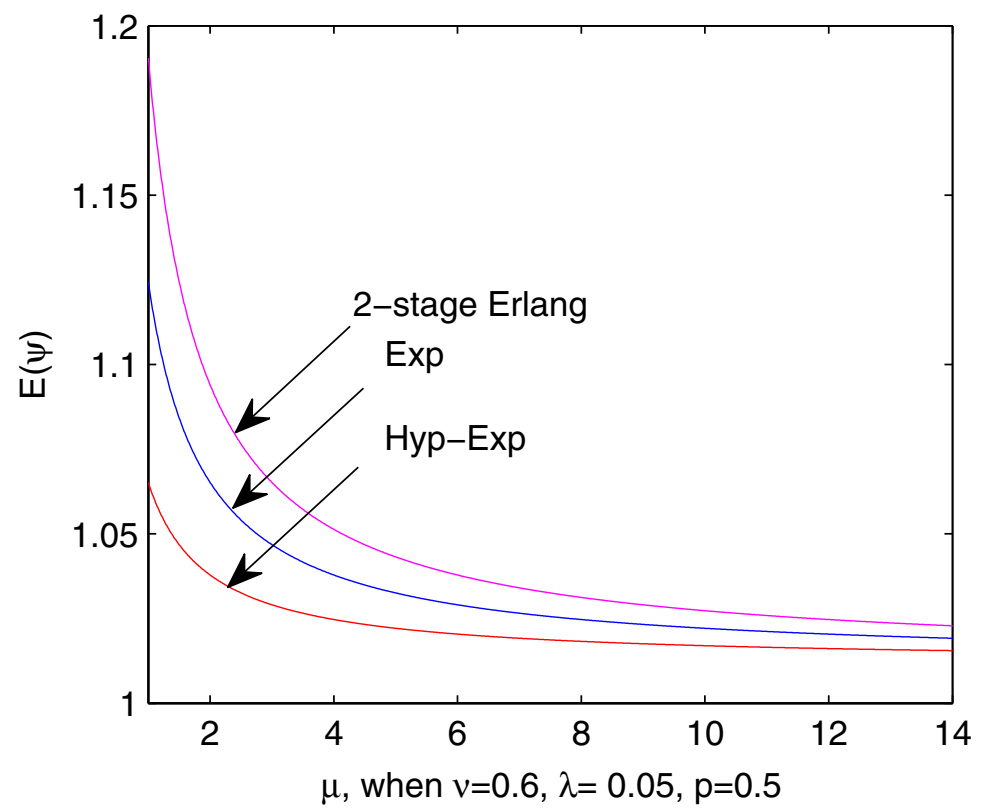

FiguRE 12. Impact of $\mu$ on $E(\psi)$ for different service time distributions.

rate $\mu$ increases the queue length decreases, which leads to increase in probability of the server is in random vacation period during a random vacation $P_{\mathrm{RV}}$ as well as in a dormant period $P_{D}$. However, we see that the hyper-exponential distribution provides highest system state probabilities $P_{\mathrm{RV}}$ and $P_{D}$ whereas 2-stage Erlang provides lowest and exponential stays in between hyper-exponential and 2-stage Erlang. It is clear from the fact that a higher coefficient of variation ( or variance) of distribution gives the higher $P_{\mathrm{RV}}$ and $P_{D}$ values. On the other hand, From Figures 12 and 13, we observe that the mean values $E(\psi)$ and $L_{\varpi}$ decreases as $\mu$ increases before it arrives stability, which is completely opposite from the Figures 10 and 11. Moreover, the averages $E(\psi)$ and $L_{\varpi}$ are larger for 2-stage Erlang service time distribution as compared to exponential and hyper-exponential distribution.

The effect of arrival rate $\lambda$ on $P_{\mathrm{RV}}, P_{D}$ and $L_{\varpi}$ for different $Y$ values is demonstrated in Figures 14-16. Here service time and vacation time follows an exponential distribution. Also, we presume that $Y$ follows a geometric distribution and the model parameters are $\mu=8, \nu=0.6$ and $p=0.5$. From Figure 14, we observe that the system state probability $P_{\mathrm{RV}}$ increases sharply in the beginning while arrival rate $\lambda$ increases and then decreases slowly for different values of $Y$. A possible explanation for this situation is as follows: for any value of $Y$, while the arrival rate $\lambda$ is comparatively small the probability of the server is in a random vacation period during a random vacation $P_{\mathrm{RV}}$ increases rapidly whereas it decreases slowly while the arrival rate $\lambda$ becomes high. The system state probability $P_{\mathrm{RV}}$ also decreases as the values of $Y$ increases, this is because as the values of $Y$ (maximum number of vacations) increases the probabilities of these $Y$ values decreases (as $Y$ follows a geometric distribution), which leads to decrease in probability of the server is in a random vacation period during a random vacation $P_{\mathrm{RV}}$. For a large value of $\lambda$, the difference between the values of $P_{\mathrm{RV}}$ is small as $Y$ increases. In Figure 15, we see that for a fixed value of $Y$, the system state probability $P_{D}$ decreases as $\lambda$ increases and for a fixed value of $\lambda$, the system state probability $P_{D}$ increases with the increasing value of $Y$. The reason is similar to Figure 14 .

The figures are useful for finding the optimal value of $Y$. It displays that the values of $P_{\mathrm{RV}}$ and $P_{D}$ for $Y=4$ and $Y$ tends to $\infty$ are very close, therefore, it may be rewarding if we select the value of $Y$ between 4 and $\infty$. Finally, From Figure 16, we observe that for a fixed value of $Y$, the average $L_{\varpi}$ increases for the increasing values of $\lambda$ as it should be. However, for a fixed value of $\lambda$, the average $L_{\varpi}$ decreases with the increasing values of $Y$. The reason is that as the values of $Y$ (maximum number of vacations) increases the probabilities of these 


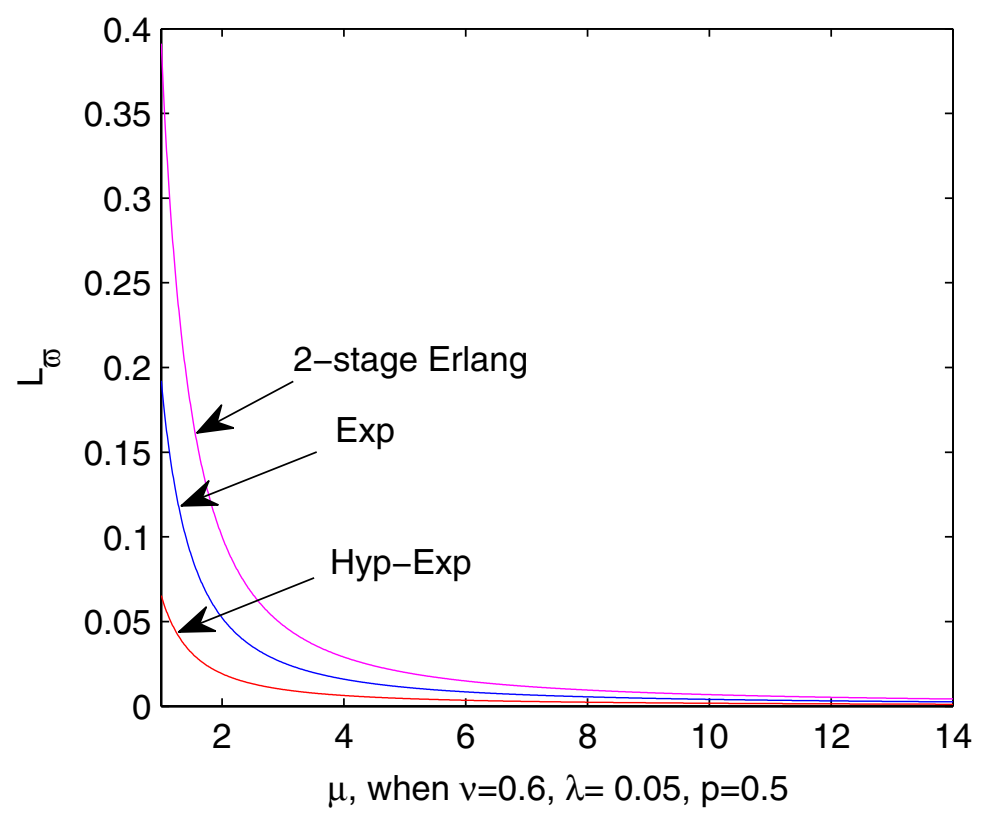

FiguRE 13. Impact of $\mu$ on $L_{\varpi}$ for different service time distributions.

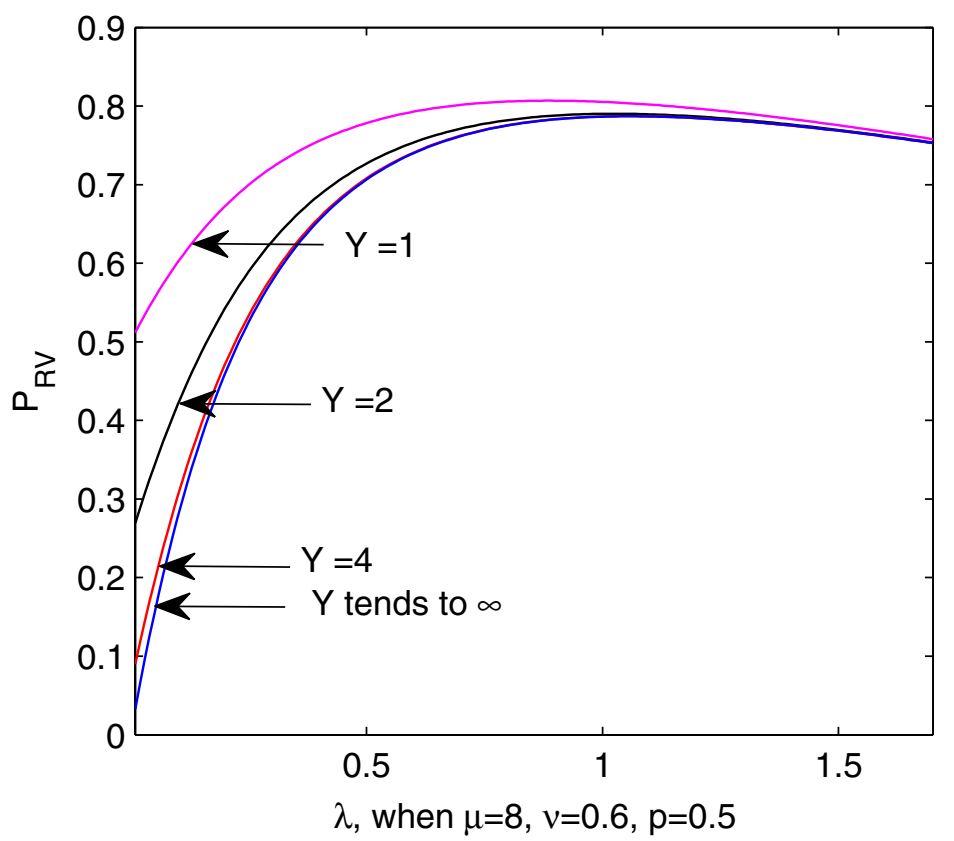

Figure 14. Impact of $\lambda$ on $P_{\mathrm{RV}}$ for different $Y$ values. 
P. KALITA AND G. CHOUDHURY

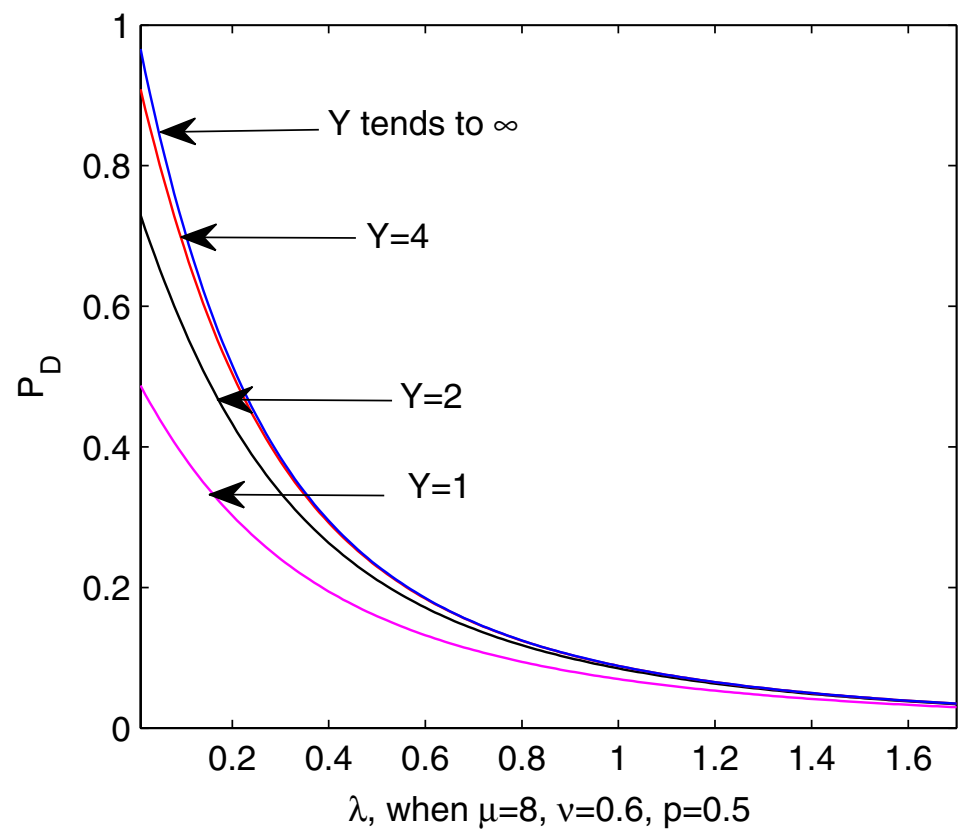

Figure 15. Impact of $\lambda$ on $P_{D}$ for different $Y$ values.

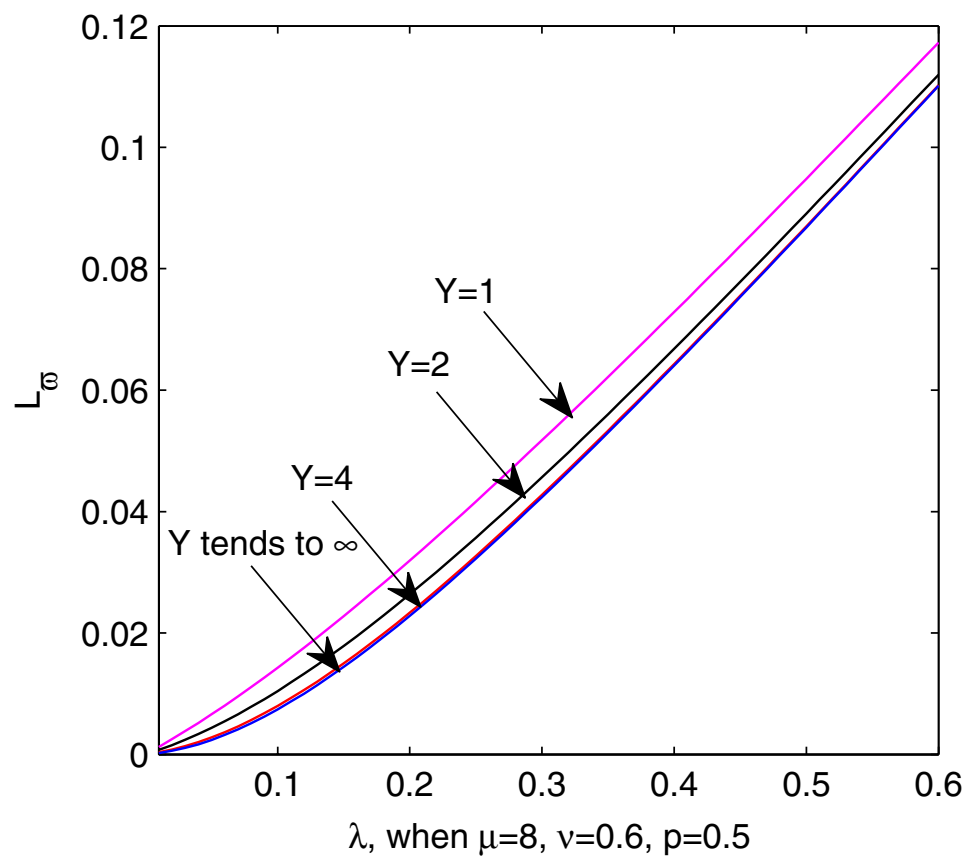

Figure 16. Impact of $\lambda$ on $L_{\varpi}$ for different $Y$ values. 
$Y$ values decreases (as $Y$ follows a geometric distribution), i.e., the probability that the server takes consecutive vacations decreases and the probability that the customers are served through a service rate increases, which gives rise to decrease in $L_{\varpi}$.

Thus, as shown in our analysis, we can conclude that the probability associated with vacations, service time distribution and different vacation policies have played a significant role. It would be interesting to investigate some other queueing systems, e.g., the proposed model can be extended to gated or limited service discipline under random vacation policy.

\section{REFERENCES}

[1] S. Alouf, E. Altman and A.P. Azad, Analysis of an $M / G / 1$ queue with repeated inhomogeneous vacations with application to IEEE 802.16e power saving mechanism. In: Proc. QEST. Saint-Malo France (2008) 27-36.

[2] G. Choudhury, A batch arrival queue with a vacation time under single vacation policy. Comput. Oper. Res. 29 (2002) 1941-1955.

[3] G. Choudhury, Analysis of the $M^{X} / G / 1$ queueing system with vacation times. Sankhya: Indian J. Stat. 64 (2002) $37-49$.

[4] R.B. Cooper, Introduction to Queueing Theory. Elsevier, Amsterdam (1981).

[5] B.T. Doshi, Queueing Systems with vacations a survey. Queueing Syst. 1 (1986) 29-66.

[6] B.T. Doshi, Single sever queues with vacations. In: Stochastic Analysis of Computer and Communication systems, edited by H. Takagi. North-Holland (1990) 217-265.

[7] S.W. Fuhrmann and R.B. Cooper, Stochastic decomposition in the $M / G / 1$ queue with generalized vacations. Oper. Res. 33 (1985) 1117-1129.

[8] D.P. Heyman, Optimal operating policies for $M / G / 1$ queueing system. Oper. Res. 16 (1968) $362-382$.

[9] S. Jeyakumar and B. Senthilnathan, Modelling and analysis of a bulk service queueing model with multiple working vacations and server breakdown. RAIRO: OR 51 (2017) 485-508.

[10] J.C. Ke, Operating characteristic analysis on the $M^{X} / G / 1$ system with a variant vacation policy and balking. Appl. Math. Model. 31 (2007) 1321-1337.

[11] J.C. Ke, K.B. Huang and W.L. Pearn, The randomized vacation policy for a batch arrival queue. Appl. Math. Model. 34 (2010) $1524-1538$.

[12] J.C. Ke, C.H. Wu and Z.G. Zhang, Recent developments in vacation queueing models: a short survey. Int. J. Oper. Res. 7 (2010) 3-8.

[13] J.C. Ke, F.M. Chang and T.H. Liu, $M / M / c$ balking retrial queue with vacation. Qual. Technol. Quant. Manage. 16 (2019) $54-66$.

[14] O. Kella, The threshold policy in the $M / G / 1$ queue with server vacations. Nav. Res. Logist. 36 (1989) 111-123.

[15] V.G. Kulkarni, Modelling and Analysis of Stochastic Systems. Chapman \& Hall, London (1995).

[16] H.S. Lee and M.M. Srinivasan, Control policies for the queueing system. Manage. Sci. 35 (1989) 708-721.

[17] H.W. Lee, S.S. Lee, J.O. Park and K.C. Chae, Analysis of $M^{X} / G / 1$ queue with $N$ policy and multiple vacations. J. Appl. Prob. 31 (1994) 467-496.

[18] S.S. Lee, H.W. Lee, S.H. Yoon and K.C. Chae, Batch arrival queue with $N$ policy and single vacation. Comput. Oper. Res. 22 (1995) 173-189.

[19] K.K. Leung, On the additional delay in an $M / G / 1$ queue with generalized vacations and exhaustive service. Oper. Res. 40 (1992) 272-283.

[20] Y. Levy and U. Yechiali, Utilization of idle time in an $M / G / 1$ queue with server vacations. Manage. Sci. 22 (1975) $202-211$.

[21] Y. Park and G.U. Hwang, Performance modeling and analysis of the sleep-mode in IEEE 802.16e WMAN. In: Proc. IEEE Vehicular Technology Conference (VTC) (2007) 2801-2806.

[22] S.M. Ross, Stochastic Process. Wiley. New York (1983).

[23] J.B. Seo, S.Q. Lee, N.H. Park, H.W. Lee and C.H. Cho, Performance analysis of sleep mode operation in IEEE802.16e. Vehi. Tech. Conf. 2 (2004) 1169-1173.

[24] L. Tadj and G. Choudhury, Optimal design and control of queues. Top 13 (2005) 359-412.

[25] L. Takacs, Introduction to the Theory of Queues. Oxford University press, New York (1962).

[26] H. Takagi, Queueing analysis. In: Vol. 1 of Vacation and Priority Systems. North Holland, Amsterdam (1991).

[27] N. Tian, Multi stage adaptive vacation policies in an queueing system. Appl. Math. 4 (1992) 12-18.

[28] N. Tian and Z.G. Zhang, Vacation Queueing Models: Theory and Applications. Springer, New York, USA (2006).

[29] R. Wolff, Poisson arrivals see time averages. Oper. Res. 30 (1982) 223-231.

[30] D.Y. Yang, M.F. Chang and J.C. Ke, On an unreliable retrial queue with general repeated attempts and $J$ optional vacations. Appl. Math. Model. 40 (2016) 3275-3288.

[31] B. Yang, Z. Hou and J. Wu, Analysis of the equilibrium strategies in the Geo/Geo/1 queue with multiple working vacations. Qual. Technol. Quant. Manage. 15 (2018) 663-685.

[32] Z.G. Zhang and N. Tian, Discrete time queue with multiple adaptive vacations. Queueing Syst. 38 (2001) $419-429$. 